\title{
miR-146a modulates autoreactive Th17 cell differentiation and regulates organ-specific autoimmunity
}

\author{
Bo Li, ${ }^{1}$ Xi Wang, ${ }^{1}$ In Young Choi, ${ }^{1}$ Yu-Chen Wang, ${ }^{1}$ Siyuan Liu, ${ }^{1}$ Alexander T. Pham, ${ }^{1}$ Heesung Moon, ${ }^{1}$ Drake J. Smith ${ }^{1}$, \\ Dinesh S. Rao, ${ }^{2,3,4,5}$ Mark P. Boldin, ${ }^{6}$ and Lili Yang ${ }^{1,2,3,4}$ \\ 1Department of Microbiology, Immunology and Molecular Cenetics, ${ }^{2}$ Eli and Edythe Broad Center of Regeneration Medicine and Stem Cell Research, ${ }^{3}$ onsson Comprehensive Cancer Center, \\ the David Geffen School of Medicine, ${ }^{4}$ Molecular Biology Institute, and ${ }^{5}$ Department of Pathology and Laboratory Medicine, UCLA, Los Angeles, California, USA. \\ ${ }^{6}$ Department of Molecular and Cellular Biology, Beckman Research Institute, City of Hope, Duarte, California, USA.
}

\begin{abstract}
Autoreactive CD4 T cells that differentiate into pathogenic Th17 cells can trigger autoimmune diseases. Therefore, investigating the regulatory network that modulates Th17 differentiation may yield important therapeutic insights. miR146a has emerged as a critical modulator of immune reactions, but its role in regulating autoreactive Th17 cells and organspecific autoimmunity remains largely unknown. Here, we have reported that miR-146a-deficient mice developed more severe experimental autoimmune encephalomyelitis (EAE), an animal model of human multiple sclerosis (MS). We bred miR-146a-deficient mice with 2D2 T cell receptor-Tg mice to generate 2D2 CD4 T cells that are deficient in miR-146a and specific for myelin oligodendrocyte glycoprotein (MOC), an autoantigen in the EAE model. miR-146a-deficient 2D2 T cells induced more severe EAE and were more prone to differentiate into Th17 cells. Microarray analysis revealed enhancements in IL-6- and IL-21-induced Th17 differentiation pathways in these T cells. Further study showed that miR-146a inhibited the production of autocrine IL- 6 and IL-21 in $2 \mathrm{D} 2$ T cells, which in turn reduced their Th17 differentiation. Thus, our study identifies miR-146a as an important molecular brake that blocks the autocrine IL-6- and IL-21-induced Th17 differentiation pathways in autoreactive CD4 T cells, highlighting its potential as a therapeutic target for treating autoimmune diseases.
\end{abstract}

\section{Introduction}

CD4 $\mathrm{T}$ cells play a pivotal role in orchestrating the reactions of the innate and adaptive immune systems. After activation, CD4 $\mathrm{T}$ cells can differentiate into distinct subsets of effector $\mathrm{T}$ helper (Th) cells such as Th1, Th2, Th17 cells, induced regulatory T cells (iTregs), and follicular B helper T cells (Tfh) (1-5). The list is still actively growing and now includes the recently characterized Th9 and Th22 cells $(6,7)$. These subsets of Th cells display distinct functions and regulate appropriate cellular and humoral immune responses to a wide range of pathogens (8). The differentiation of Th cells thus needs to be tightly regulated to allow for effective immune protection while avoiding inflammation-induced pathogenesis (9-11). In particular, Th17 cells characterized by their production of IL-17 family proinflammatory cytokines are important effector cells in host defense against certain fungi and extracellular bacteria; however, if Th17 cells are induced inappropriately, they can act as potent stimulators of autoimmune diseases such as multiple sclerosis (MS) and ulcerative colitis (UC) $(12,13)$. Understanding the molecular control of Th17 differentiation thus holds the key to utilizing the host defense capacity of Th17 cells, while preventing their pathogenic autoimmune effects. Several

Authorship note: B. Li and X. Wang contributed equally to this work. Conflict of interest: The authors have declared that no conflict of interest exists. Submitted: March 15, 2017; Accepted: July 18, 2017.

Reference information: J Clin Invest. 2017;127(10):3702-3716.

https://doi.org/10.1172/JCI94012. key molecular pathways involved in Th17 cell differentiation have been identified, including the classical IL-6/TGF- $\beta /$ ROR $\gamma$ t pathway, the alternative IL-21/TGF- $\beta$ /ROR $\gamma$ t pathway, and the IL-23/ IL-22 amplification pathway $(12,13)$. These discoveries provide important insights into the molecular network that regulates Th17 cell differentiation, but the whole picture of this critical regulatory network has just begun to be revealed.

miRs are a family of small, noncoding RNAs that negatively regulate gene expression by binding to the 3'-UTR region of their target genes $(14,15)$. Over the past decade, miRs have emerged as a novel class of posttranscriptional regulators that modulate various aspects of immune cell biology, ranging from developmental to effector functions $(16,17)$. In particular, miR-146a has been identified as a critical negative regulator of immune reactions (18). About a decade ago, in an effort to screen for miRs involved in the innate immune cell response to microbial infection, $3 \mathrm{miRs}$ ( $m i R-132, m i R-155$, and $m i R-146 a)$ were identified that showed strong upregulation upon bacterial LPS stimulation (19). Among them, miR-146a attracted special attention because of its NF-кBdependent induction and its targeted attenuation of the NF- $\mathrm{B}$ signaling transducers TRAF6 and IRAK1 (19). The physiological significance of miR-146a was evidenced by the phenotype of miR146a-deficient mice $(20,21)$. These mice developed a chronic hyperinflammatory and autoimmune disorder that was correlated with constitutive activation of $\mathrm{NF}-\mathrm{\kappa B}$ signaling and characterized by a gradual accumulation of hyperproliferating myeloid cells, activated CD4 and CD8 T cells, and autoantibodies $(20,21)$. 
Study of the autoimmune symptoms in the miR-146a-deficient mice revealed a deficiency of natural regulatory T cells (nTregs) in these mice that failed to control Th1 proinflammatory responses and was probably caused by a dysregulated IFN- $\gamma$ signaling pathway (22). Our study of miR-146a deficiency in conventional T cells showed that loss of miR-146a caused a hyperresponsiveness of $\mathrm{T}$ cells in both acute antigenic responses and chronic inflammatory autoimmune responses (23). These findings highlight miR-146a as a potent inhibitor of $\mathrm{T}$ cell-mediated autoimmunity. However, the role of miR-146 in directly regulating autoreactive $\mathrm{T}$ cell activities, in particular that of regulating the differentiation of autoreactive CD4 T cells into pathogenic Th17 cells that are potent triggers of organ-specific autoimmune diseases, remains largely unknown.

This study focuses on determining how miR-146a regulates autoreactive CD4 T cell activities in mouse experimental autoimmune encephalomyelitis (EAE), an animal model of human MS (24). We found that miR-146a-deficient mice developed a more severe EAE that featured an exaggerated Th17 response against autoantigen. 2D2 $\mathrm{T}$ cell receptor-transgenic (TCR-Tg) mice (referred to hereafter as 2D2-Tg mice) produce clonal CD4 T cells recognizing the myelin oligodendrocyte glycoprotein (MOG) that is an autoantigen in the EAE disease model (25). By breeding miR146a-deficient mice with 2D2 TCR-Tg mice, we generated autoreactive 2D2 CD4 T cells that are specific for MOG and deficient in miR-146a. Adoptive transfer of miR-146a-deficient 2D2 T cells into RAG1-deficient recipient mice induced more severe EAE that was associated with an enhanced Th17 response. In vitro analysis showed that, compared with their WT counterparts, miR-146adeficient 2D2 T cells produced more proinflammatory cytokines, in particular IL-17A, and were more prone to differentiate into Th17 cells. Microarray analysis revealed an enhanced Th17 differentiation network in miR-146a-deficient 2D2 T cells, especially the pathways that are induced by IL- 6 and IL-21. Study of the IL-6Th17 and IL-21-Th17 differentiation pathways showed that miR146a-deficient 2D2 $\mathrm{T}$ cells produced higher levels of autocrine IL- 6 and IL-21 that contributed to their enhanced Th17 responses. We further proved that TRAF6 and IRAK1 are bona fide targets of miR-146a in 2D2 T cells and are possible mediators for regulating the Th17 differentiation of these autoreactive CD4 T cells, at least partly through modulation of their NF- $\kappa \mathrm{B}$ activation-induced production of autocrine IL-6 and IL-21. Notably, autocrine IL-21 can be further induced by the Th17 cell master transcription factor ROR $\gamma$ t once ROR $\gamma \mathrm{t}$ is upregulated in differentiated Th17 cells. Therefore, we believe that our study identifies miR-146a as a new and important molecular brake that blocks the autocrine IL-6/ IL-21 Th17 differentiation pathways in autoreactive CD4 T cells and controls T cell-mediated autoimmunity.

\section{Results}

miR-146a-deficient mice develop more severe EAE featuring an exaggerated Th17 response against autoantigen. We induced EAE in WT and miR-146a-deficient mice (denoted as $m i R-146 a^{-/-}$mice) by treating the mice with $\mathrm{MOG}_{35-55} / \mathrm{CFA}$ emulsion and pertussis toxin (PTX) following a standard protocol (Figure 1A, and see Methods). $\mathrm{MOG}_{35-55}$ is the myelin oligodendrocyte glycoprotein peptide that serves as the CD4 T cell-responsive autoantigen in this EAE disease model (25). Compared with the WT control mice, $m i R-146 a^{-/-}$ mice developed more severe EAE, evidenced by their higher EAE clinical scores (Figure 1B), as well as increased infiltration of lymphocytes into their spinal cords (Figure 1, C and D). When stimulated with the $\mathrm{MOG}_{35-55}$ autoantigen, splenic cells harvested from the $m i R-146^{-/}$EAE mice produced significantly higher levels of the Th17 cytokine IL-17A, while their production levels of the Th1 cytokine IFN- $\gamma$ and the Th2 cytokine IL- 4 were similar to those detected in WT control splenic cells (Figure 1E). Analysis of spinal cord-infiltrating CD4 T cells identified higher numbers of IL-17A-producing cells in the $m i R-146 a^{-/}$EAE mice (Figure 1, F and G). These IL-17A ${ }^{+}$ CD4 T cells coproduced high levels of IFN- $\gamma$ and granulocyte macrophage-CSF (GM-CSF), a signature of pathogenic Th17 cells in the EAE model (Supplemental Figure 1, A and B; supplemental material available online with this article; https://doi.org/10.1172/ JCI94012DS1). Analysis of spinal cord-infiltrating CD4 T cells harvested from WT EAE mice also revealed an upregulation of miR146a expression in these cells that peaked 2 weeks after EAE induction (Figure 1H). Therefore, miR-146a upregulation in autoreactive CD4 T cells is associated with EAE disease progress in mice, while $m i R-146^{-/-}$mice develop more severe EAE featuring an exaggerated Th17 response against autoantigen.

miR-146a-deficient autoreactive CD4 $T$ cells induce a more severe EAE that is associated with enhanced Th17 responses against autoantigen. By breeding 2D2-Tg mice with $m i R-146 a^{-/-}$mice, we generated 2D2-Tg mice deficient in miR-146a (referred to hereafter as 2D2/miR-146a $a^{-/-}$mice). 2D2 is a Tg CD4 TCR that recognizes $\mathrm{MOG}_{35-55}$; therefore, CD4 T cells harvested from 2D2-Tg mice are specific for the $\mathrm{MOG}_{35-55}$ autoantigen and can be considered autoreactive $\mathrm{T}$ cells and used to induce EAE (25). We isolated naive CD4 $\mathrm{T}$ cells from $2 \mathrm{D} 2-\mathrm{Tg}$ mice or from $2 \mathrm{D} 2 / \mathrm{miR}-146 \mathrm{a}^{-/-}$ mice (referred to hereafter as 2D2 or 2D2-KO T cells, respectively), adoptively transferred these T cells into RAG1-deficient mice (referred to hereafter as $R A G 1^{-/-}$mice), and then induced EAE in the $R A G 1^{-/-}$recipient mice (Figure $2 \mathrm{~A}$ ). This adoptive transfer EAE model allowed us to study the possible role of miR-146a as an autologous factor regulating autoreactive CD4 $\mathrm{T}$ cells for induction of EAE. As shown in Figure 2B, $R A G 1^{-/-}$mice receiving 2D2-KO T cells developed more severe EAE than did the control mice receiving 2D2 T cells. To study the functionality of the adoptively transferred 2D2-KO T cells, splenic cells harvested from $R A G 1^{-1-}$ EAE mice were stimulated with PMA and ionomycin followed by intracellular cytokine staining. Compared with 2D2 T cells, 2D2-KO T cells were more prone to differentiate into Th17 cells that produced IL-17A (Figure 2, C and D; 2D2 and 2D2-KO $\mathrm{T}$ cells were gated as $\mathrm{TCR} \beta^{+} \mathrm{CD} 4^{+}$cells). In agreement with the intracellular cytokine staining results we obtained, when stimulated with the $\mathrm{MOG}_{35-55}$ autoantigen, splenic cells from $R A G 1^{-/-}$ EAE mice receiving 2D2-KO T cells produced higher levels of IL-17A as measured by ELISA (Figure 2E). On the other hand, production of IFN- $\gamma$ and IL- 4 was not significantly different between the 2 groups of splenic cells (Figure 2E). Therefore, in the EAE disease model, miR-146a deficiency predisposes autoreactive CD4 T cells to develop into pathologic Th17 cells and promotes T cell-mediated autoimmunity.

miR-146a-deficient autoreactive CD4 $T$ cells show altered Th cytokine production. To study the role of miR-146a in regulating cytokine production of autoreactive CD $4 \mathrm{~T}$ cells, we isolated naive 

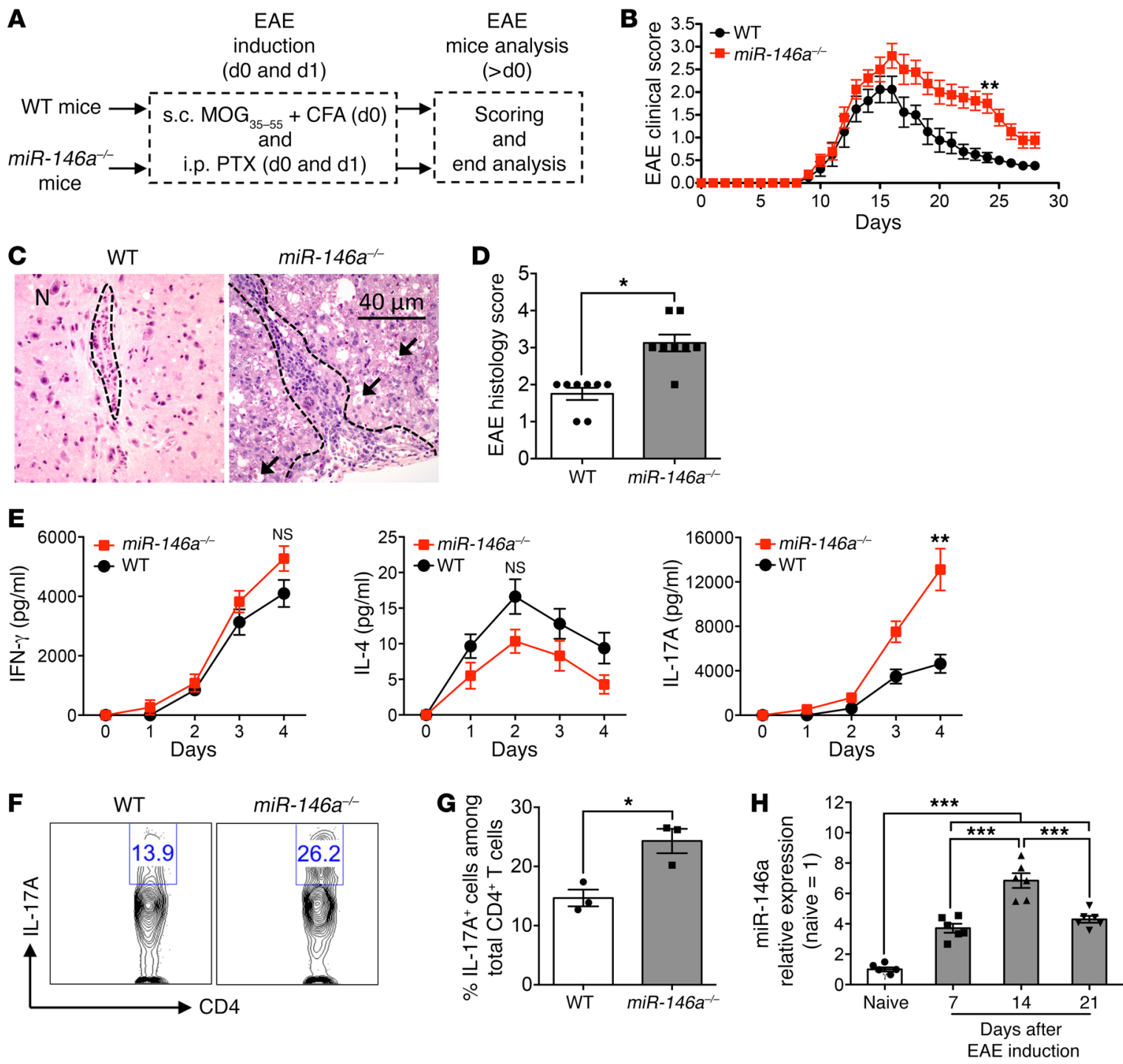

Figure 1. miR-146a-deficient mice develop more severe experimental EAE featuring exaggerated Th17 responses against autoantigen. The experiments were repeated 3 times, and representative results are presented. (A) Schematic representation of the experimental design to induce EAE in WT and miR$146 a^{-1-}$ mice. (B) EAE clinical scores for experimental mice over the time course. Data are presented as the mean \pm SEM $(n=8)$. ${ }^{* *} P<0.01$, by Student's $t$ test. (C) Representative histological images showing H\&E-stained spinal cord sections from day-28 EAE mice $(n=8)$. Note that there was more inflammation (primarily perivascular and lymphocytic, shown in the areas within the dashed lines) in the spinal cords of miR-146a ${ }^{-/-}$mice. Arrows indicate degenerating axons. Scale bar: $40 \mu \mathrm{m}$. (D) Quantification of the H\&E-stained spinal cord sections presented in C. Data are presented as the mean \pm SEM $(n=8)$. ${ }^{*} P<0.05$, by Student's $t$ test. (E) ELISA analysis of cytokine production by splenic cells harvested from day-28 EAE mice and stimulated with MOG $_{35-55}$. Data are presented as the mean \pm SEM of triplicate cultures. ${ }^{* *} P<0.01$, by Student's $t$ test. (F) Representative FACS plots showing the intracellular IL-17A staining of spinal cord-infiltrating lymphocytes (pregated on TCR $\beta^{+} C D 4^{+}$cells) harvested from day-18 EAE mice $(n=3)$. (G) Quantification of the FACS plots presented in $\mathbf{F}$. Data are presented as the mean $\pm \operatorname{SEM}(n=3)$. ${ }^{*} P<0.05$, by Student's $t$ test. (H) qPCR analysis of miR-146a expression in spinal cord-infiltrating $\mathrm{CD}^{+}{ }^{+}$T cells harvested from WT mice at the indicated time points after EAE induction. Naive, WT mice prior to EAE induction. Data are presented as the mean $\pm \operatorname{SEM}(n=6) .{ }^{* *} P<0.001$, by 1 -way ANOVA.

2D2 or 2D2-KO T cells from 2D2-Tg and 2D $2 / m i R-146 a^{-/-}$mice and stimulated these cells in vitro using anti-CD3 and anti-CD28 (see Methods). Compared with 2D2 T cells, 2D2-KO T cells showed a significantly altered Th cytokine production profile. These cells increased their production of the Th17 cytokine IL-17A as well as the Th1 cytokine IFN- $\gamma$, but decreased their production of the Th2 cytokine IL-4, at both the gene expression and protein production levels (Figure 3, A and B). Thus, miR-146a differentially regulates the production of proinflammatory Th1/Th17 and antiinflammatory Th2 cytokines by autoreactive CD 4 T cells.
miR-146a deficiency promotes the in vitro differentiation of autoreactive CD4 T cells into Th17 cells. To study the expression of miR146a during Th differentiation of autoreactive CD4 T cells, we cultured 2D2 T cells in vitro under various Th-polarizing conditions, and then examined their miR-146a expression using TaqMan quantitative PCR (qPCR). Both the IL-6-induced and IL-21induced Th17 polarization conditions [denoted as Th17 (+IL-6) and Th17 (+IL-21), respectively] were included to investigate the possible roles of miR-146a in the classical and alternative Th17 differentiation pathways $(12,13)$. We observed a significant increase 

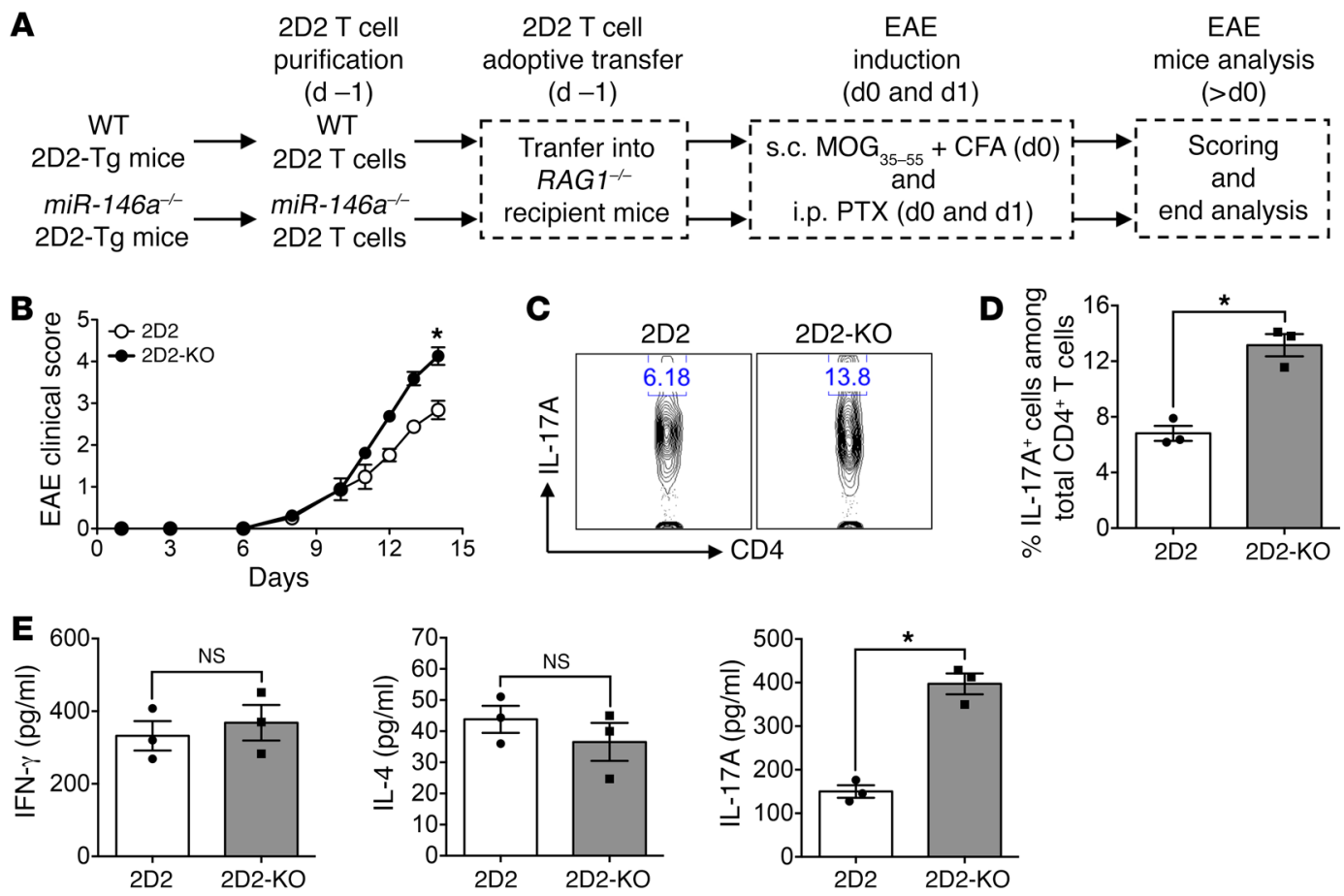

Figure 2. miR-146a-deficient autoreactive CD4 T cells induce a more severe EAE that is associated with enhanced Th17 responses against autoantigen. The experiments were repeated 3 times, and representative results are presented. (A) Schematic representation of the experimental design to study the role of miR-146a as an autonomous factor regulating autoreactive CD4 T cell induction of EAE in the RAG1/- mouse adoptive transfer model. (B) EAE clinical scores for the 2 groups of RAG1-/- mice adoptively transferred with either WT or miR-146a-- $2 \mathrm{D} 2 \mathrm{~T}$ cells (denoted as $2 \mathrm{D} 2$ or $2 \mathrm{D} 2-\mathrm{KO}$ mice, respectively) over the time course. Data are presented as the mean $\pm \operatorname{SEM}(n=8) .{ }^{*} P<0.05$, by Student's $t$ test. (C) Representative FACS plots showing intracellular IL-17A staining of $2 \mathrm{D} 2$ and $2 \mathrm{D2}-\mathrm{KO}$ T cells (pregated as TCR $\beta^{+} \mathrm{CD} 4^{+}$) harvested from day-14 EAE mice $(n=3)$. (D) Quantification of the FACS plots presented in C. Data are presented as the mean $\pm \operatorname{SEM}(n=3) .{ }^{*} P<0.05$, by Student's $t$ test. (E) ELISA analysis of cytokine production by splenic cells harvested from day-14 EAE mice and stimulated with $\mathrm{MOC}_{35-55}$. Irradiated $\mathrm{B} 6$ splenic cells were provided as antigen-presenting cells. Data are presented as the mean \pm $\operatorname{SEM}(n=3) .{ }^{*} P<0.05$, by Student's $t$ test.

in miR-146a expression in effector 2D2 $\mathrm{T}$ cells compared with that in naive 2D2 $\mathrm{T}$ cells (denoted as Tn cells), under all culture conditions (Figure 4A). In particular, Th17-polarized 2D2 T cells expressed high levels of miR-146a, suggesting a possible role of miR-146a in regulating Th17 differentiation. Indeed, when we cultured 2D2 and 2D2-KO T cells under Th17-polarizing conditions, 2D2-KO T cells were more prone to differentiate into Th17 cells, as measured by intracellular IL-17A cytokine staining (Figure 4, B and C). Gene expression analysis confirmed that 2D2-KO T cells expressed an elevated level of the IL-17A effector cytokine gene, as well as the gene encoding RORyt that is considered to be the master transcription factor driving the differentiation of Th17 cells (Figure 4D) (26). Thus, miR-146a deficiency promotes the Th17 differentiation of autoreactive CD4 T cells.

Microarray analysis reveals enhanced IL-6/IL-21-Th17 differentiation pathways in miR-146a-deficient autoreactive CD4 T cells. In order to identify the key molecular pathways involved in promoting the Th17 differentiation of miR-146a-deficient autoreactive $\mathrm{CD} 4 \mathrm{~T}$ cells, we compared the gene expression profiles of $2 \mathrm{D} 2$ and 2D2-KO effector T cells. Naive 2D2 and 2D2-KO T cells were stimulated in vitro with anti-CD3 and anti-CD28 for 3 days. On day 3, the resulting 2D2 and 2D2-KO effector T cells were collected, and total RNA was isolated for whole-genome gene expression profile analysis using microarray technology. As shown in Figure 5A, genome-wide analysis revealed differential expression of a signif- icant portion of genes between 2D2 and 2D2-KO effector T cells. Comparative analysis of selected Th1, Th2, and Th17 lineagespecific genes revealed differential expression of many Th lineage-specific genes between the 2D2 and 2D2-KO effector T cells (Figure 5B). In particular, multiple genes involved in inducing Th17 differentiation were upregulated in the 2D2-KO effector T cells, especially the genes in the IL-6-mediated Th17 induction pathway (Figure 5B) $(27,28)$. During Th17 differentiation, IL-6 activates STAT3, which collaborates with the SMADs activated by TGF- $\beta$ to induce expression of ROR $\gamma$ t, the master transcription factor of Th17 differentiation, which then induces expression of Th17 effector cytokines such as IL-17A and IL-21 (Figure 5D) (12, 13). Our microarray data revealed an upregulation of almost all genes, including IL-6, STAT3, ROR $\gamma$ t, IL-17A, and IL-21, involved in the IL-6-mediated Th17 induction pathway, a finding that was further confirmed by qPCR analysis (Figure $5, \mathrm{~B}-\mathrm{D}$ ). This is an interesting observation, because IL-6 is generally considered to be an environmental factor that influences Th17 differentiation, while in this case, IL- 6 was produced by the autoreactive CD4 T cells themselves. It was also interesting to find that IL-21 was upregulated in 2D2-KO T cells. IL-21 is considered the key cytokine responsible for inducing the alternative Th17 differentiation pathway $(12,13)$. The observed overexpression of IL-21 in miR-146a-deficient CD4 $\mathrm{T}$ cells suggests that these $\mathrm{T}$ cells may benefit from an enhanced alternative pathway when differentiating into Th17 cells. There- 

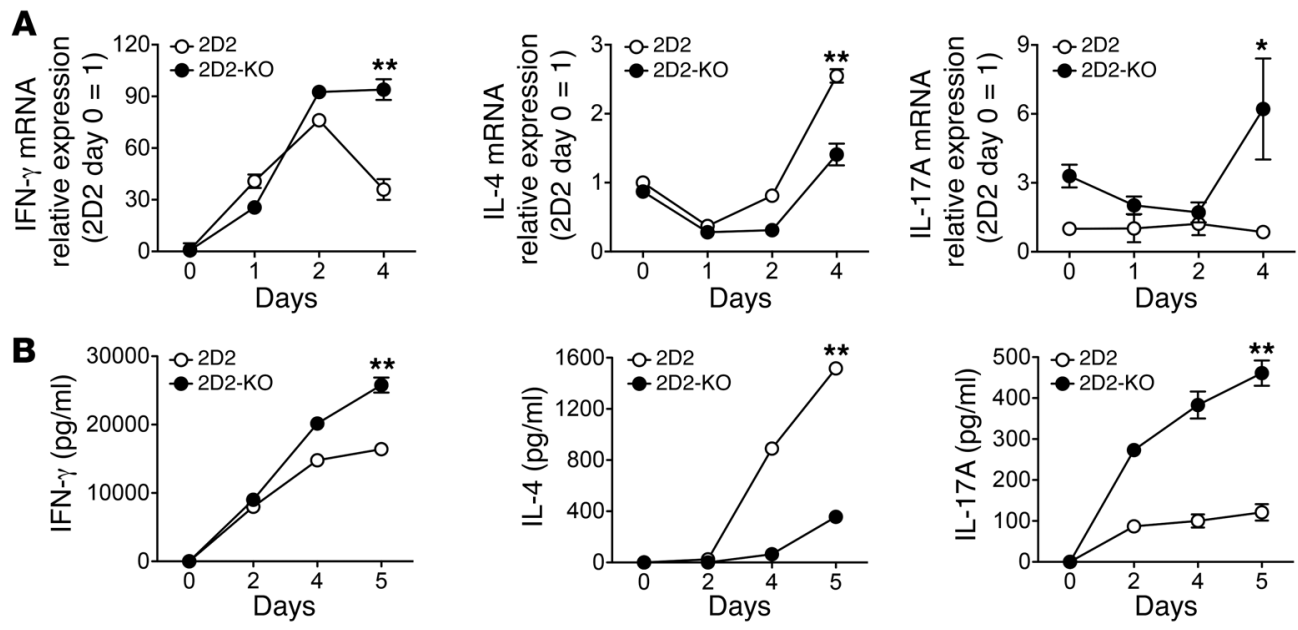

Figure 3. miR-146a-deficient autoreactive CD4 T cells show altered Th cytokine production. Naive CD4 T cells were sorted from WT and miR-146adeficient 2D2-Tg mice (denoted as $2 \mathrm{D2}$ and 2D2-KO T cells, respectively) and cultured in vitro for 5 days in the presence of plate-bound anti-CD3 (5 $\mu \mathrm{g} / \mathrm{ml})$ and soluble anti-CD28 $(1 \mu \mathrm{g} / \mathrm{ml})$. Over the time course, cells and cell culture supernatants were collected for analysis. The experiments were repeated more than 3 times, and representative results are presented. (A) qPCR analysis of Th cytokine mRNA expression in $2 D 2$ and 2D2-KO T cells. Data are presented as the mean \pm SEM of triplicate cultures. ${ }^{*} P<0.05$ and ${ }^{* *} P<0.01$, by Student's $t$ test. (B) ELISA analysis of Th cytokine levels in $2 \mathrm{D} 2$ and $2 \mathrm{D2}-\mathrm{KO} \mathrm{T}$ cell culture supernatants. Data are presented as the mean \pm SEM of triplicate cultures. ${ }^{* *} P<0.01$, by Student's $t$ test.

fore, our microarray study reveals enhanced IL-6/IL-21 Th17 differentiation pathways in miR-146a-deficient autoreactive CD4 T cells and suggests a possible role for T cell autocrine IL- 6 and IL-21 in mediating miR-146a regulation of Th17 polarization of autoreactive CD4 T cells.

miR-146 a deficiency predisposes autoreactive CD4 T cells to Th17 responses through modulation of $T$ cell autocrine $I L-6$ and $I L-21$. To assess the possible roles of T cell autocrine IL- 6 and IL-21 in modulating Th17 polarization of miR-146a-deficient autoreactive CD4 $\mathrm{T}$ cells, we stimulated $2 \mathrm{D} 2$ and $2 \mathrm{D} 2-\mathrm{KO} \mathrm{T}$ cells in vitro and analyzed their production of IL- 6 and IL-21. Compared with 2D2 T cells, 2D2-KO T cells produced significantly more IL-6 and IL-21 at both the gene expression and cytokine secretion levels (Figure 6, A and B). Accordingly, compared with that seen in 2D2 T cells, TCR stimulation in 2D2-KO T cells induced stronger STAT3 phosphorylation (p-STAT3), which could be further amplified by additional exogenous IL-6 or IL-21 (Figure 6, C and D). Notably, IL-6-STAT3 forms a positive feedback loop, because once activated, the p-STAT3 dimer can bind to the IL- 6 gene promoter and induce the production of more IL-6 cytokine as well as the IL-6 receptor signaling subunit gp130 (29). To directly study how T cell autocrine IL-6 and IL-21 may affect the Th17 response of 2D2-KO $\mathrm{T}$ cells, we blocked IL-6 and/or IL-21 signaling in 2D2-KO T cell cultures by adding to the cultures neutralizing antibodies against IL-6 and/or IL-21. As shown in Figure 6, E and F, either IL-6- or IL-21-neutralizing antibody blockage alone significantly reduced IL-17A gene expression and protein production in 2D2-KO T cells, while the combination of IL- 6 and IL-21 blockage showed a synergistic effect and further reduced IL-17A gene expression and protein production. A detailed analysis of the IL-6/IL-21 signaling pathways in 2D2-KO T cells compared with those in 2D2 T cells revealed an overexpression of IL-6 and IL-21 receptors (IL-6R $\alpha$, gp130, and IL-21R $\alpha$ ), as well as a reduction of the IL-6 receptor signaling inhibitors SOCS3 and p-SHP-2 (Supplemental Figure 5,
B-D). SOCS3 works through inhibition of Jak1 activation, while SHP-2 acts via dephosphorylation of the IL-6 receptor signaling subunit gp130 (29). Of note, production levels of the soluble IL-6R $\alpha($ sIL-6R $\alpha)$ that has been indicated in the induction of IL-6 "trans-signaling" did not change, suggesting that sIL-6R $\alpha$ is not likely involved in the miR-146a regulation of IL-6 signaling in autoreactive CD4 T cells (Supplemental Figure 5A). Taken together, these results indicate that miR-146a deficiency predisposes autoreactive CD4 T cells to the Th17 response, at least partially through modulation of T cell autocrine IL- 6 and IL-21.

TRAF6 and IRAK1 are bona fide targets of miR-146a in autoreactive CD4 $T$ cells and are possible mediators of $m i R-146 a$ in its regulation of Th17 differentiation of autoreactive CD4 T cells. miRs function through posttranscriptional repression of their target genes $(14,15)$. We have previously identified TRAF6 and IRAK1, two adaptor molecules involved in the NF- $\kappa \mathrm{B}$ activation pathway, as targets of miR-146a in mouse $\mathrm{T}$ cells that mediate miR-146a control of the resolution of $\mathrm{T}$ cell acute and chronic inflammatory responses $(23,30)$. To study whether TRAF6 and IRAK1 are also the targets of miR-146a in regulating autoreactive CD4 $\mathrm{T}$ cell Th17 differentiation, we compared the protein levels of TRAF6 and IRAK1 in 2D2 and 2D2-KO T cells and found that TRAF6 and IRAK1 protein levels were increased in the 2D2-KO T cells (Figure 7, A and B). On the other hand, overexpression of miR-146a in 2D2 $\mathrm{T}$ cells through transduction of $2 \mathrm{D} 2 \mathrm{~T}$ cells with miR-induced gene silencing-miR-146a (MIG-miR-146a) retroviral vector significantly reduced the protein levels of TRAF6 and IRAK1 (Figure 7, C and D). Thus, TRAF6 and IRAK1 are bona fide targets of miR-146a in autoreactive CD4 T cells.

Next, we studied how miR-146a may affect NF-kB activity in autoreactive CD4 T cells. Compared with 2D2 T cells, miR-146adeficient 2D2-KO T cells induced stronger NF- $\mathrm{BB}$ activation after TCR stimulation, in particular the activation of NF- $\kappa B$ subunit p65 and cRel (Figure 7E). On the contrary, overexpression of miR- 
A

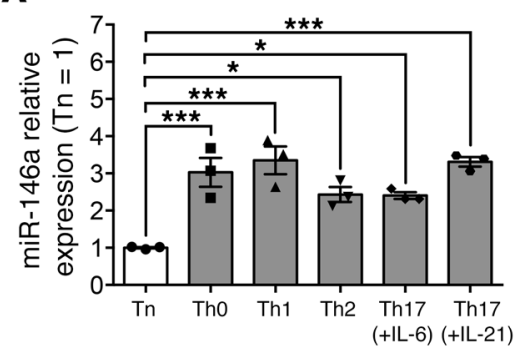

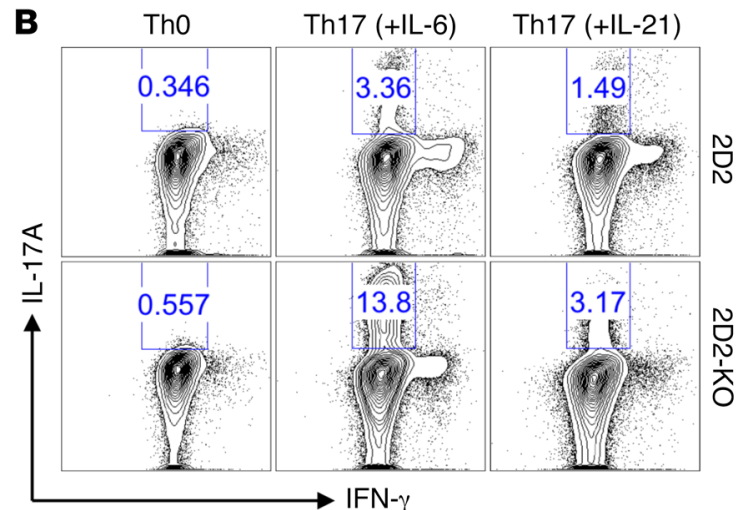
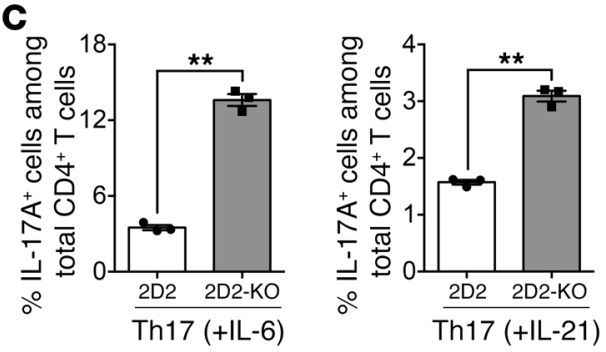

D

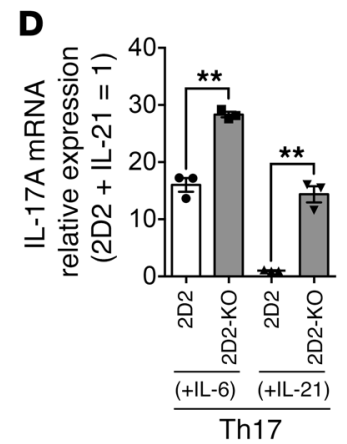

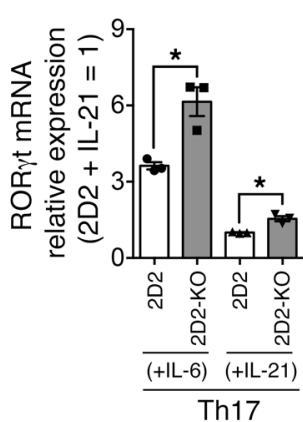

Figure 4. miR-146a deficiency promotes autoreactive CD4 T cells to differentiate into Th17 cells. Naive CD4 T cells were sorted from WT and miR146a-deficient 2D2-Tg mice (denoted as $2 \mathrm{D} 2$ and 2D2-KO T cells, respectively) and stimulated for 4 days with plate-bound anti-CD3 (5 $\mu \mathrm{g} / \mathrm{ml})$ and soluble anti-CD28 $(1 \mu \mathrm{g} / \mathrm{ml})$, under either nonpolarizing (Th0) or various Th-polarizing (Th1, Th2, or Th17) conditions. On day 4, cells were collected for analysis. The experiments were repeated 3 times, and representative results are presented. (A) qPCR analysis of miR-146a expression in $2 D 2 \mathrm{~T}$ cells cultured under the indicated conditions. Data are presented as the mean \pm SEM of triplicate cultures. ${ }^{*} P<0.05$ and ${ }^{* * *} P<0.001$, by 1 -way ANOVA. (B) Representative FACS plots showing intracellular IL-17A and IFN- $\gamma$ staining of $2 \mathrm{D} 2$ and $2 \mathrm{D2}-\mathrm{KO}$ T cells cultured under the indicated conditions (pregated as TCR $\beta^{+} \mathrm{CD} 4^{+}$cells). (C) Quantification of the FACS plots presented in B. Data are presented as the mean \pm SEM of triplicate cultures. ${ }^{* *} P<0.01$, by Student's $t$ test. (D) qPCR analysis of IL-17A and ROR $\gamma \mathrm{t}$ mRNA expression in $2 \mathrm{D2}$ and 2D2-KO T cells cultured under the indicated Th17-polarizing conditions. Data are presented as the mean \pm SEM of triplicate cultures. ${ }^{*} P<0.05$ and ${ }^{* *} P<0.01$, by Student's $t$ test.

146a in 2D2 T cells reduced NF- $\mathrm{KB}$ p65 and cRel activation in the MIG-miR-146a-transduced $\mathrm{T}$ cells after TCR stimulation (Figure 7F). NF-kB p65 and cRel are strong inducers of IL-6 and IL-21. Accordingly, an elevated activation of NF- $\mathrm{KB}$ in $2 \mathrm{D} 2-\mathrm{KO} \mathrm{T}$ cells is associated with an elevated production of IL- 6 and IL-21 in these $\mathrm{T}$ cells (Figure 6, A and B). On the other hand, blocking NF- $\mathrm{kB}$ activation in 2D2 and 2D2-KO T cells by adding the NF- $\mathrm{kB}$ inhibitor Bay 11-7082 to the $\mathrm{T}$ cell culture abrogated the production and eliminated the differential expression of IL- 6 and IL-21 in 2D2 and 2D2-KO T cells (Figure 7G). Hence, miR-146a modulates NF-kB activity and the resulting production of IL-6/IL-21 in autoreactive CD4 T cells, probably by targeting TRAF6 and IRAK1.

To study how the increased levels of TRAF6 and IRAK1 in miR146a-deficient autoreactive CD4 T cells may impact their Th17 differentiation, we knocked down TRAF6 and/or IRAK1 gene expression in 2D2-KO T cells using siRNAs and examined Th17 signaling and differentiation of the siRNA-transfected T cells (Figure 8, A-G). Our analysis of siRNA-transfected 2D2-KO T cells for STAT3 phosphorylation (Figure 8A), autocrine IL-6 and IL-21 production levels (Figure 8, B and C), and ROR $\gamma$ t and IL-17A gene expression (Figure $8, \mathrm{D}$ and $\mathrm{E}$ ) revealed that knocking down the gene expression of either TRAF6 or IRAK moderately reduced IL-6/IL-21/Th17 pathway signaling events, while knocking down the expression of both
TRAF6 and IRAK genes showed a significant reduction of these signaling events. To directly assay their capacity to differentiate into Th17 cells, we cultured the siRNA-transfected 2D2-KO T cells under Th17-polarizing conditions. When both TRAF6 and IRAK1 were knocked down in 2D2-KO T cells, the addition of only exogenous IL- 6 did not rescue their Th17 differentiation, probably due to the presence of an autocrine IL-21 Th17 differentiation pathway (Figure 8, F and G). Indeed, when exogenous IL-21 was added on top of exogenous IL-6, the Th17 differentiation of siTRAF6- and siIRAK1-transduced 2D2-KO T cells was rescued (Figure 8, F and G). These data suggest that TRAF6 and IRAK1 act synergistically to mediate miR-146a regulation of Th17 differentiation of autoreactive $\mathrm{CD} 4 \mathrm{~T}$ cells, probably through modulating their production of autocrine IL- 6 and IL-21.

Taken together, our results indicate that TRAF6 and IRAK1 are bona fide targets of miR-146a in autoreactive CD4 $\mathrm{T}$ cells and are possible mediators of miR-146a in its regulation of Th17 differentiation of autoreactive $\mathrm{CD} 4 \mathrm{~T}$ cells, at least partially through modulation of the T cell autocrine IL-6/IL-21 Th17 differentiation pathway.

\section{Discussion}

On the basis of our study, we proposed an autocrine IL-6/IL-21 blockade model. In this model, miR-146a inhibits the differen- 
A
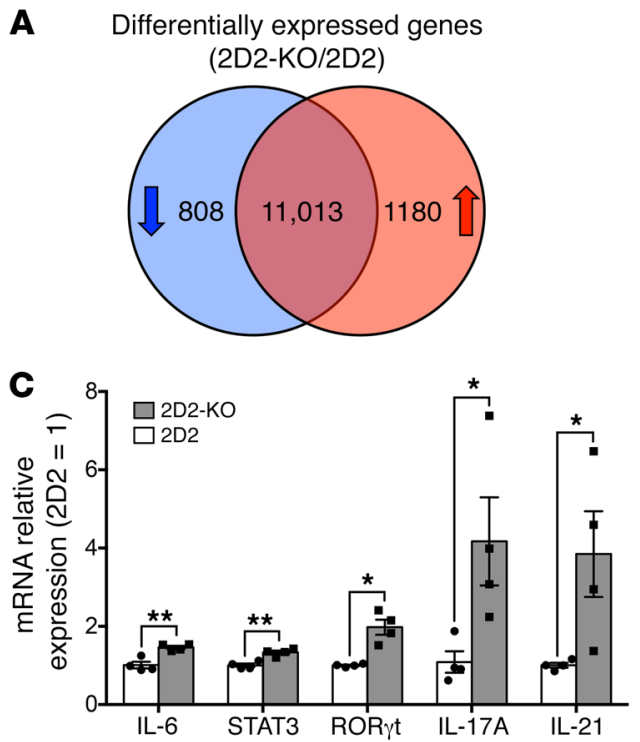

D

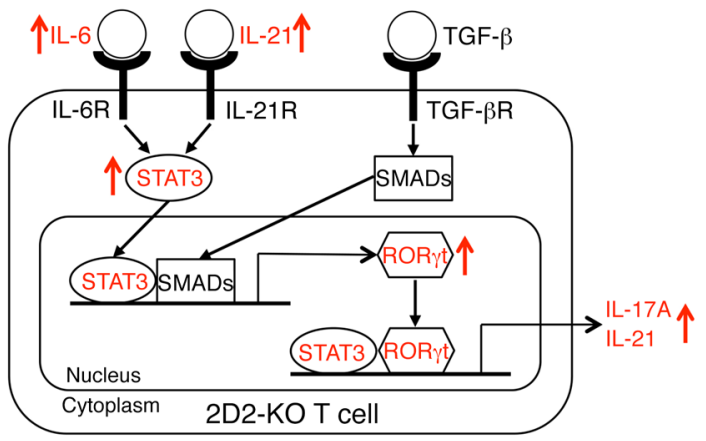

B

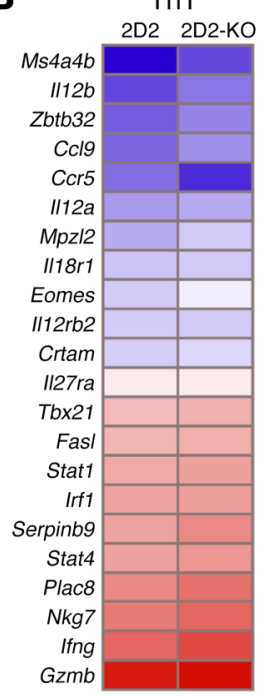

Th2

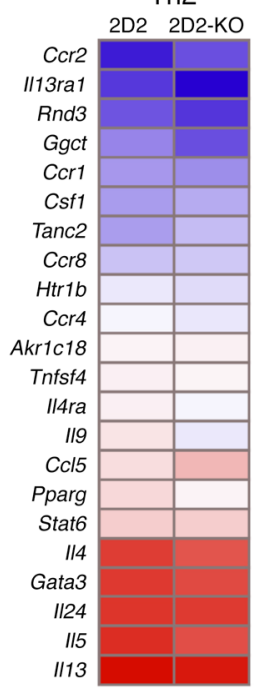

Th17

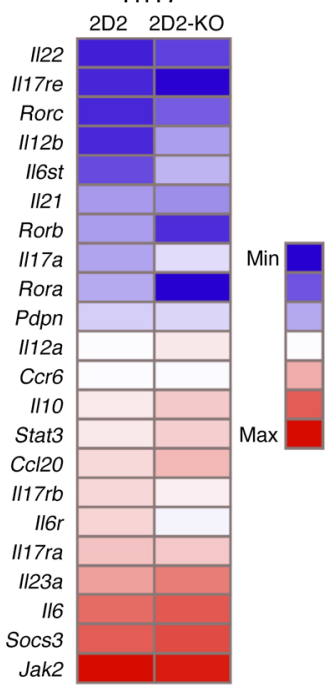

Figure 5. Microarray analysis reveals enhanced IL-6/IL-21 Th17 differentiation pathways in miR-146a-deficient autoreactive CD4 T cells. (A and B) Naive CD4 T cells were sorted from WT and miR-146a-deficient 2D2-Tg mice (denoted as 2D2 and 2D2-KO T cells, respectively) and stimulated for 3 days with plate-bound anti-CD3 $(5 \mu \mathrm{g} / \mathrm{ml})$ and soluble anti-CD28 $(1 \mu \mathrm{g} / \mathrm{ml})$. On day 3, cells were collected, and total RNA was isolated for whole-genome gene expression profile analysis using the GeneChip Mouse Genome 4302.0 arrays ( $n=2$ mice per group). (A) Venn diagram of the differentially expressed genes in $2 \mathrm{D} 2$ and $2 \mathrm{D2}$-KO T cells. Genes showing altered expression with a $P$ value of less than 0.05 and greater than 1.5 -fold changes were considered differentially expressed. (B) Heatmaps showing the expression of selected Th1, Th2, and Th17 lineage-specific genes in 2D2 and 2D2-KO T cells. (C) qPCR validation of the mRNA expression of selected IL-6/IL-21 Th17 differentiation pathway genes identified from the microarray analysis. Naive 2D2 and 2D2-K0 T cells were isolated and stimulated for 3 days with anti-CD3 and anti-CD28, following the same procedure as that used for the microarray study. On day 3 , total RNA was isolated for gene expression analysis using QPCR. The experiments were repeated 3 times. Representative data are presented as the mean \pm SEM of quadruplicate cultures. ${ }^{*} P<0.05$ and ${ }^{* *} P<0.01$, by Student's $t$ test. (D) Schematic showing the molecular signaling pathways regulating IL-6/ IL-21-induced Th17 differentiation. Differentially expressed genes identified by microarray and validated by qPCR are highlighted in red.

tiation of autoreactive CD4 T cells into pathogenic Th17 cells by blocking the T cell autocrine IL-6/IL-21 Th17 differentiation pathway (Figure 9). Upon recognition of autoantigens, autoreactive CD4 T cells initiate TCR signaling that leads to NF- $\mathrm{KB}$ activation. NF- $\mathrm{kB}$ induces the production of T cell autocrine IL-6 and IL-21 cytokines that activate STAT3. STAT3 induces the expression of the master transcription factor gene governing Th17 cell differentiation, ROR $\gamma$ t, which then induces the expression of Th17 effector cytokines like IL-17A and IL-21. Notably, autocrine IL-21 can be induced by both the initial TCR/NF- $\mathrm{kB}$ signaling and the subsequent Th17/ROR $\gamma t$ signaling. Therefore, T cell autocrine IL- 6 and IL-21 promote the differentiation of autoreactive $\mathrm{CD} 4 \mathrm{~T}$ cells into pathogenic Th17 cells. As a negative feedback regulator of NF- $\mathrm{\kappa B}$ signaling, miR-146a is induced by NF- $\mathrm{kB}$, which in turn down-

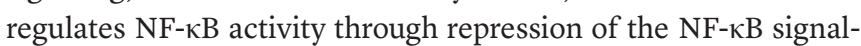

ing transducers TRAF6 and IRAK1. Through downregulation of NF- $\kappa B$ activity, miR-146a blocks the $\mathrm{T}$ cell autocrine IL-6/IL-21 Th17 differentiation pathway in autoreactive CD4 T cells and inhibits their development into pathogenic Th17 cells.

Many molecular pathways have been implicated in regulating the generation of Th17 cells: IL- 6 plus TGF- $\beta$ together induce ROR $\gamma \mathrm{t}$ and the Th17 transcriptional program (the classical pathway); IL-21 is produced by Th17 cells themselves and, together with TGF- $\beta$, is able to induce Th17 differentiation (the alternative pathway); IL-23 is not the differentiation factor of Th17 cells, but stabilizes differentiating Th17 cells and leads to the further maturation of Th17 cells (12, 27, 28, 31-34). Through regulation of IL-6 and IL-21 production, miR-146a can be placed within both the classical IL-6/TGF- $\beta$ / ROR $\gamma \mathrm{t}$-mediated Th17 differentiation pathway and the alternative IL-21/TGF- $\beta /$ ROR $\gamma$ t-mediated Th17 differentiation pathway. The 
A

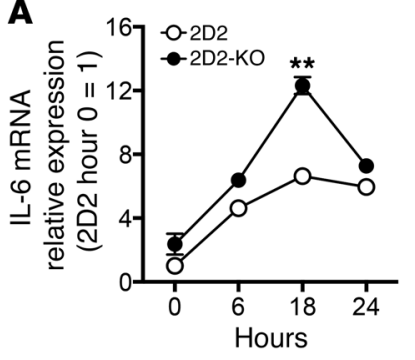

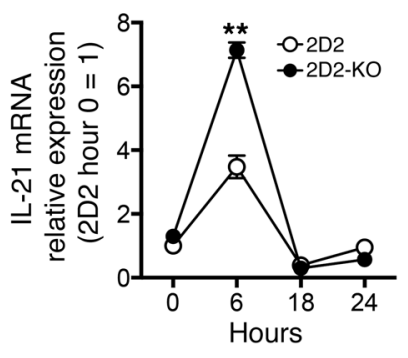

B
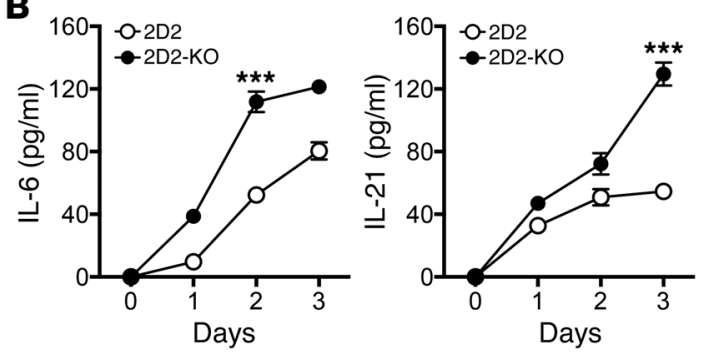

C

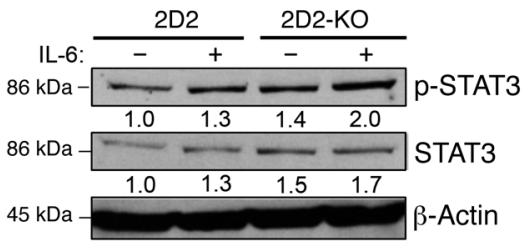

D

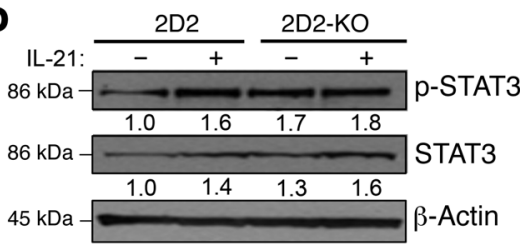

E

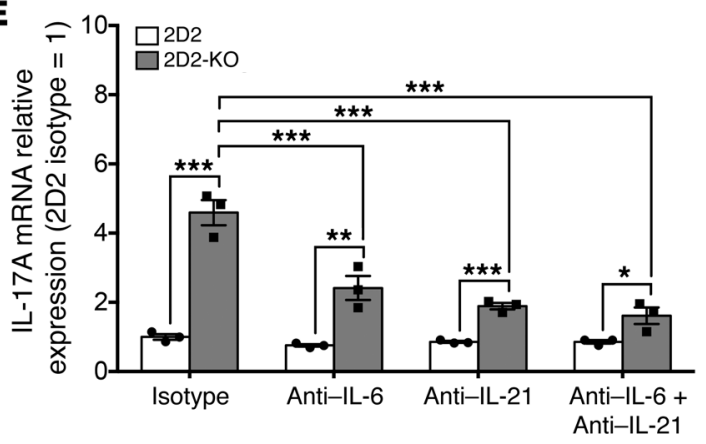

$\mathbf{F}$

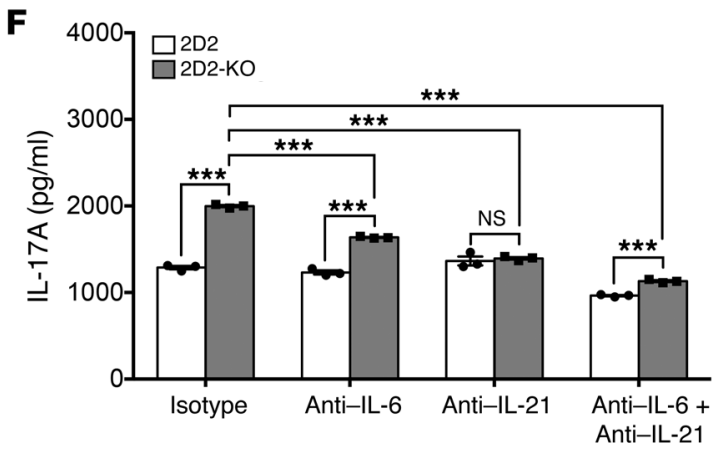

Figure 6. miR-146a deficiency predisposes autoreactive CD4 T cells to Th17 responses through modulation of T cell autocrine IL-6 and IL-21. The experiments were repeated 3 times, and representative results are presented. (A) Time-course qPCR analysis of IL-6 and IL-21 mRNA expression in $2 D 2$ and 2D2-KO T cells stimulated with plate-bound anti-CD3 $(5 \mu \mathrm{g} / \mathrm{ml})$ and soluble anti-CD28 $(1 \mu \mathrm{g} / \mathrm{ml})$. Data are presented as the mean \pm SEM of triplicate cultures. ${ }^{* *} P<0.01$, by Student's $t$ test. (B) Time-course ELISA analysis of IL-6 and IL-21 cytokine production by $2 \mathrm{D} 2$ and $2 \mathrm{D} 2-\mathrm{KO} \mathrm{T}$ cells stimulated with plate-bound anti-CD3 $(5 \mu \mathrm{g} / \mathrm{ml})$ and soluble anti-CD28 $(1 \mu \mathrm{g} / \mathrm{ml})$. Data are presented as the mean \pm SEM of triplicate cultures. ${ }^{* * *} P<0.001$, by Student's $t$ test. (C and D) Western blot analysis of p-STAT3 and STAT3 protein levels in 2D2 and 2D2-KO T cells. Prior to analysis, 2D2 and 2D2-KO T cells were stimulated with plate-bound anti-CD3 $(5 \mu \mathrm{g} / \mathrm{ml})$ and soluble anti-CD28 $(1 \mu \mathrm{g} / \mathrm{ml})$ for 2 days and then further stimulated for 10 minutes, with or without the addition of exogenous IL-6 $(50 \mathrm{ng} / \mathrm{ml})(\mathbf{C})$ or IL-21 $(100 \mathrm{ng} / \mathrm{ml})(\mathbf{D})$. (E and F) qPCR analysis of IL-17A mRNA expression (E) and ELISA analysis of IL-17A protein production (F). 2D2 and 2D2-KO T cells were stimulated with plate-bound anti-CD3 $(5 \mu \mathrm{g} / \mathrm{ml})$ and soluble anti-CD28 $(1 \mu \mathrm{g} / \mathrm{ml})$ for 3 days in the presence or absence of anti-IL-6-neutralizing $(10 \mu \mathrm{g} / \mathrm{ml})$ or anti-IL-21-neutralizing $(10 \mu \mathrm{g} / \mathrm{ml})$ antibodies or isotype control antibodies (10 $\mu \mathrm{g} / \mathrm{ml})$. On day 3 , cells were collected for qPCR analysis, while cell culture supernatants were collected for ELISA analysis. Data are presented as the mean \pm SEM of triplicate cultures. ${ }^{*} P<0.05$, ${ }^{* *} P<0.01$, and ${ }^{* *} P<0.001$, by Student's $t$ test for pairwise comparisons within each treatment group and by 2 -way ANOVA for multiple comparisons between different treatment groups.

critical role of IL- 6 in inducing Th17 cells and autoimmune diseases is evidenced by the phenotype of IL-6-deficient mice, which have a defect in their ability to generate Th17 cells and are resistant to EAE and antigen-induced arthritis (31, 35-38). However, IL-6 is traditionally considered to be a tissue inflammatory factor that is produced during the acute-phase response to inflammation by cells of the innate immune system such as DCs, monocytes, macrophages, mast cells, and B cells, and also by nonimmune cells such as tumor cells, fibroblasts, endothelial cells, and keratinocytes (39-41). Our study is the first to our knowledge to suggest that autocrine IL-6 produced by autoreactive CD4 T cells also plays an important role in polarizing these cells toward Th17 differentiation and that $\mathrm{T}$ cell-autonomous miR-146a is a critical molecular brake blocking these cells from producing autocrine IL- 6 and differentiating into pathogenic Th17 cells. On the other hand, IL-21 is generally considered an effector cytokine produced by committed Th17 cells that acts in a positive feedback loop to further promote Th17 responses $(12,13)$. We believe our study is the first to demonstrate that CD4 $\mathrm{T}$ cell-autonomous miR-146a negatively regulates TCR-induced IL-21 expression and IL-21-induced Th17 differentiation, probably through inhibition of TCR/NF- $\mathrm{BB}$ cRel signaling (Figure 7, E and F).

In addition to IL-17A, miR-146a-deficient autoreactive CD4 T cells also showed altered production of other Th cytokines. Overall, miR-146a deficiency promotes the production of proinflammatory Th1 and Th17 cytokines (e.g., IFN- $\gamma$ and IL-17A) and suppresses the production of antiinflammatory Th2 cytokines (e.g., IL-4) (Figure 3, A and B). Because miR-146a primarily targets TCR/ $\mathrm{NF}-\kappa \mathrm{B}$ signaling in autoreactive CD4 $\mathrm{T}$ cells, we asked whether miR-146a regulation of Th cytokine production is related to NF- $\kappa B$ signaling. Also, because miR-146a-deficient autoreactive CD4 T cells produce excess levels of autocrine IL-6, which is considered a major early-phase amplifier of inflammation responses, we asked 

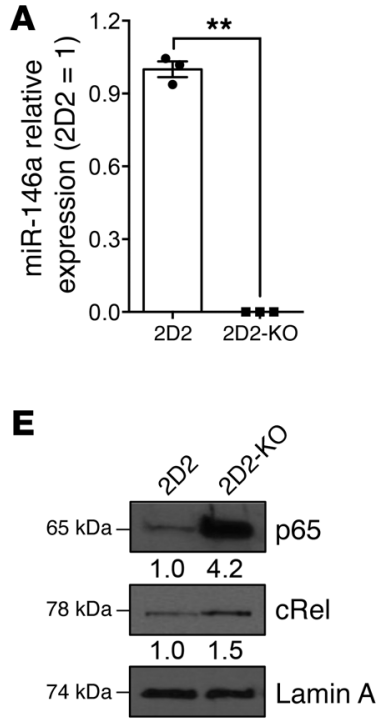
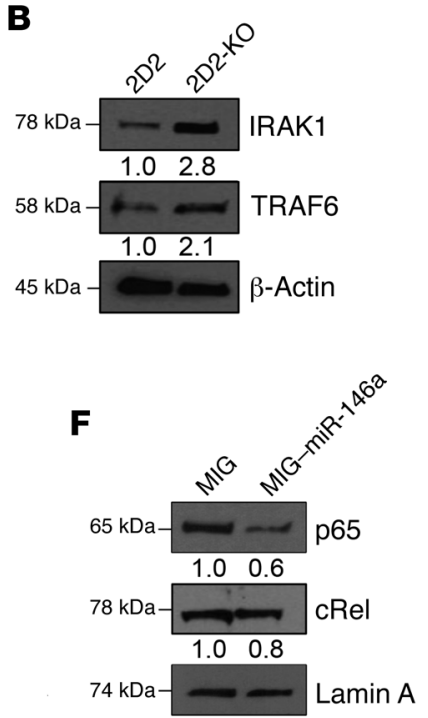
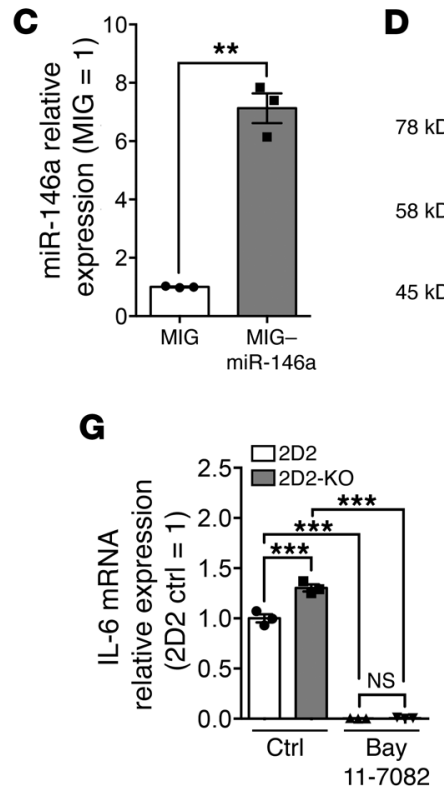
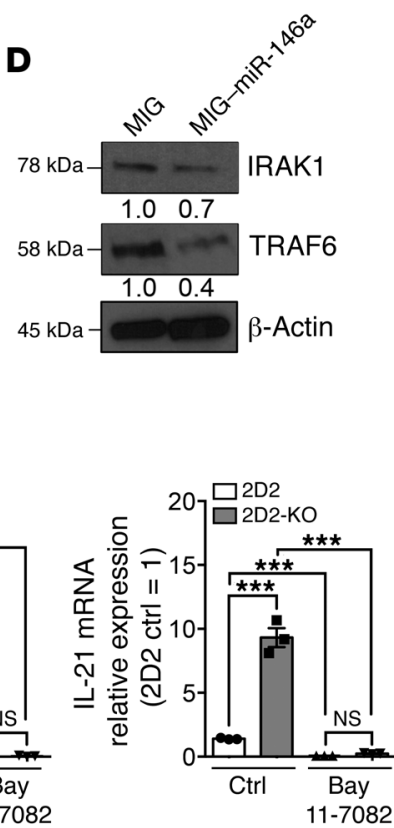

Figure 7. TRAF6 and IRAK1 are bona fide targets of miR-146a in autoreactive CD4 $\mathbf{T}$ cells. The experiments were repeated 3 times, and representative results are presented. (A and B) $2 \mathrm{D} 2$ and $2 \mathrm{D} 2-\mathrm{KO}$ T cells were stimulated for 4 days with plate-bound anti-CD3 $(5 \mu \mathrm{g} / \mathrm{ml})$ and soluble anti-CD28 $(1 \mu \mathrm{g} / \mathrm{ml})$. On day 4, the $2 \mathrm{D2}$ and $2 \mathrm{D2}-\mathrm{KO}$ T cells were collected for analysis. (A) qPCR analysis of miR-146a expression. Data are presented as the mean $\pm \mathrm{SEM}$ of triplicate cultures. ${ }^{* *} P<0.01$, by Student's $t$ test. (B) Western blot analysis of TRAF6 and IRAK1 protein levels. (C and $\left.\mathbf{D}\right) 2 \mathrm{D} 2 \mathrm{~T}$ cells were stimulated with plate-bound anti-CD3 $(5 \mu \mathrm{g} / \mathrm{ml})$ and soluble anti-CD28 $(1 \mu \mathrm{g} / \mathrm{ml})$ for 4 days. On days 2 and 3, cells were spin infected with MIG-miR-146a or control MIC retroviral vectors. On day 4, the transduced $2 \mathrm{D} 2 \mathrm{~T}$ cells were collected for analysis. (C) qPCR analysis of miR-146a expression. Data are presented as the mean \pm SEM of triplicate cultures. ${ }^{* *} P<0.01$, by Student's $t$ test. (D) Western blot analysis of TRAF6 and IRAK1 protein levels. (E) Western blot analysis of nuclear NF-KB p65 and cRel protein levels in $2 \mathrm{D} 2$ and 2D2-KO T cells stimulated with plate-bound anti-CD3 (5 $\mu \mathrm{g} / \mathrm{ml})$ and soluble anti-CD28 ( $1 \mu \mathrm{g} / \mathrm{ml})$ for 2 hours. Note that the cRel blot shows duplicate samples run on a parallel gel. (F) Western blot analysis of nuclear NF- $\kappa B$ p 65 and cRel protein levels in $2 \mathrm{D} 2 \mathrm{~T}$ cells transduced with MIG-miR-146a or control MIC retroviral vectors. $2 \mathrm{D} 2 \mathrm{~T}$ cells were stimulated with plate-bound anti-CD3 ( $5 \mu \mathrm{g} / \mathrm{ml})$ and soluble anti-CD28 $(1 \mu \mathrm{g} / \mathrm{ml})$ for 4 days. On days 2 and 3, cells were spin infected with MIG-miR-146a or control MIG retroviral vectors. On day 4, the cells were collected and rested at $4^{\circ} \mathrm{C}$ for 1 hour and then restimulated for 30 minutes with plate-bound anti-CD3 (5 $\left.\mu \mathrm{g} / \mathrm{ml}\right)$ and soluble anti-CD28 (1 $\left.\mu \mathrm{g} / \mathrm{ml}\right)$, followed by Western blotting. Note that the cRel blot shows duplicate samples run on a parallel gel. (C) qPCR analysis of IL-6 and IL-21 mRNA expression in $2 \mathrm{D2}$ and 2D2-KO T cells stimulated for 3 days with plate-bound anti-CD3 $(5 \mu \mathrm{g} / \mathrm{ml})$ and soluble anti-CD28 $(1 \mu \mathrm{g} / \mathrm{ml})$, with or without addition of the NF- $\mathrm{KB}$ inhibitor Bay 11-7082. Data are presented as the mean \pm SEM of triplicate cultures. ${ }^{* *} P<0.001$, by 1-way ANOVA. Ctrl, control.

whether $\mathrm{T}$ cell autocrine IL-6 plays a role in mediating the miR146 regulation of various Th responses. We therefore conducted experiments in which we cultured 2D2 and 2D2-KO T cells either with the addition of anti-IL-6-neutralizing antibody to block IL-6 signaling, or with the addition of the NF- $\mathrm{B}$ inhibitor Bay 11-7082 to block NF- $\mathrm{kB}$ signaling (Supplemental Figure 3). Blocking IL-6 signaling reduced the production of IFN- $\gamma$ and IL-17A, while increasing the production of IL-4 in both 2D2 and 2D2-KO T cells. For IL-17A production, blocking IL-6 signaling also significantly (Supplemental Figure 3) reduced the differences between the 2D2 and 2D2-KO T cells. These results suggest that miR-146a-regulated Th differentiation is partially mediated through the regulation of T cell autocrine IL-6. On the other hand, blocking of NF- $\kappa \mathrm{B}$ signaling largely abolished the production of all 3 Th effector cytokines (IFN- $\gamma$, IL-4, and IL-17A) in both 2D2 and 2D2-KO T cells, indicating that miR-146a-regulated Th cytokine production and Th cell differentiation are dependent on NF- $\kappa$ B signaling.

Several other miRs have been implicated in the regulation of Th17 differentiation: miR-326 promotes Th17 cell development by targeting the Th17 differentiation negative regulator Ets-1 (42); miR-10a inhibits Th17 cell differentiation indirectly by targeting B cell lymphoma 6 (Bcl6) or nuclear receptor corepressor 1 (Ncor1) and modulating T-bet expression and Th1/Th17 balance (43); miR155 promotes Th17 cell differentiation both as a $\mathrm{T}$ cell-intrinsic factor and through regulating the production of Th17 cell-polarizing cytokines by DCs $(44,45)$; and miR-301a enhances Th17 cell development, possibly through targeting protein inhibitor of activated STAT3 protein 3 (Pias3), which inhibits STAT3 signaling and Th17 cell differentiation (46). Together with our study, these findings suggest the importance of miRs in regulating Th17 cell differentiation and encourage the search for new miR candidates that contribute to the Th17 cell differentiation regulatory network (47).

An attractive feature of miRs is that a single miR can target multiple molecules and regulate multiple signaling pathways, which then collectively regulate the same or related physiological functions $(14,15)$. In this study, we show that TRAF6 and IRAK1 are bona fide targets of miR-146a in autoreactive CD4 T cells and that TRAF6 and IRAK1 jointly regulate TCR-induced NF- $\mathrm{BB}$ signaling in autoreactive CD4 T cells and modulate their IL-6/IL-21 production and Th17 differentiation (Figures 7 and 8). Besides TRAF6 and IRAK1, other verified molecular targets of miR-146a in T cells include STAT1 and protein kinase C $\epsilon$ (PRKC $\varepsilon)(48,49)$. STAT1 is involved in the IFN- $\gamma$ receptor signaling pathway and positively regulates Th1 cell differentiation, while PRKC $\varepsilon$ phos- 
A

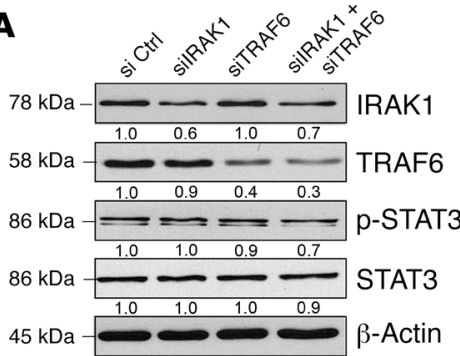

D

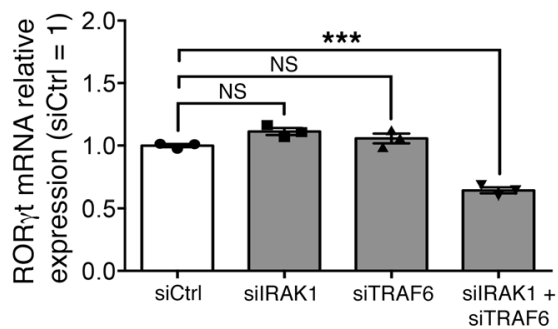

B

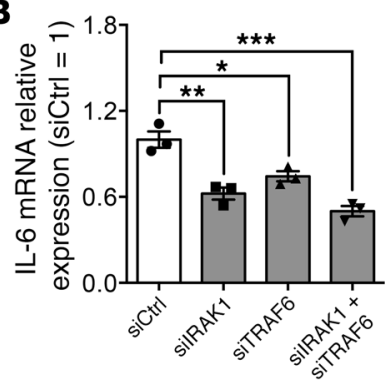

C

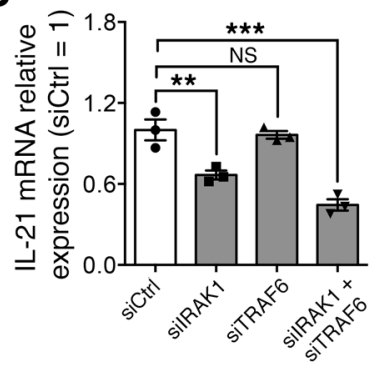

E

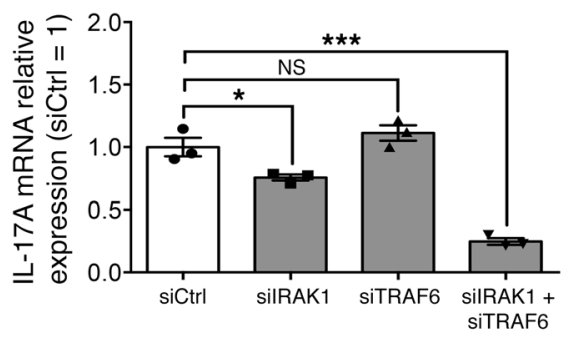

SilRAK $1+$

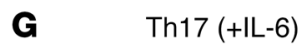

Th17 (+IL-6+IL-21)

$\mathbf{F}$

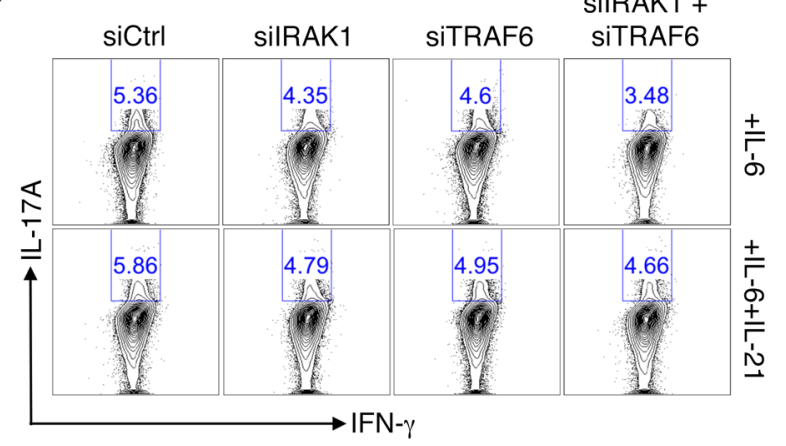

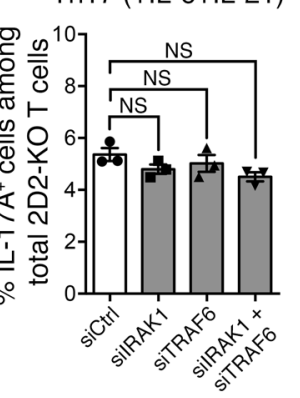

Figure 8. TRAF6 and IRAK1 are possible mediators of miR-146a-regulated Th17 differentiation of autoreactive CD4 T cells. All experiments were repeated 3 times, and representative results are presented. (A-E) 2D2-KO T cells were stimulated for 4 days with plate-bound anti-CD3 (5 $\mu \mathrm{g} / \mathrm{mI})$ and soluble anti-CD28 $(1 \mu \mathrm{g} / \mathrm{ml}$ ) and transfected with nonsilencing control siRNA (siCtrl) or siRNAs specific for mouse IRAK1 (silRAK1) or mouse TRAF6 (siTRAF6), or both (silRAK1 + siTRAF6). On day 4, cells were collected for analysis. (A) Western blot analysis of the indicated protein levels in siRNA-transfected 2D2-KO T cells. (B-E) qPCR analysis of IL-6 (B), IL-21 (C), ROR $\gamma \mathrm{t}$ (D), and IL-17A (E) mRNA expression in siRNA-transfected 2D2-KO T cells. Data are presented as the mean \pm SEM of triplicate cultures. ${ }^{*} P<0.05$, ${ }^{* *} P<0.01$, and ${ }^{* * *} P<0.001$, by 1-way ANOVA. (F and $\left.\mathbf{G}\right) 2 \mathrm{D} 2-\mathrm{KO}$ T cells were stimulated with plate-bound anti-CD3 $(5 \mu \mathrm{g} / \mathrm{ml})$ and soluble anti-CD28 $(1 \mu \mathrm{g} / \mathrm{ml})$ and transfected with the indicated siRNAs for 4 days under the indicated Th17-polarizing culture conditions. On day 4, cells were pulsed with PMA plus ionomycin for 4 hours in the presence of GolgiStop, followed by intracellular cytokine staining. (F) Representative FACS plots showing intracellular IL-17A and IFN- $\gamma$ staining of siRNA-transfected 2D2-KO T cells. (G) Quantification of the FACS plots presented in F. Data are presented as the mean \pm SEM of triplicate cultures. ${ }^{* *} P<0.01$, by 1 -way ANOVA.

phorylates STAT4, which in turn promotes Th1 cell differentiation (5). When we cultured 2D2 and 2D2-KO T cells under the Th1 differentiation condition in vitro, we found that 2D2-KO cells were more prone to differentiate into Th1 cells that produced IFN- $\gamma$ and expressed the Th1 master transcription factor T-bet (Supplemental Figure 4, A-C) (5). This observation is consistent with the enhanced Th1 proinflammatory responses reported in miR-146adeficient mice and may be mediated through miR-146a targeting of STAT1 (22). Interestingly, it has been suggested that the Th17 and Th1 differentiation pathways mutually antagonize each other $(12,13)$. In agreement with this notion, when we cultured 2D2 T cells under Th17 polarization conditions, we detected increased levels of Th17 cells (gated as IL-17A+IFN- $\gamma^{-}$) while decreased levels of Th1 cells (gated as IL-17A-IFN- $\gamma^{+}$) when the 2D2 T cells were
miR-146a deficient (Figure 4, B and C, and Supplemental Figure 4, D and E). Therefore, via targeting of multiple molecules like TRAF6, IRAK1, and STAT1, miR-146a can regulate a network of signaling pathways that collectively prevent autoreactive CD4 T cells from mounting proinflammatory Th1 and Th17 responses and inducing $\mathrm{T}$ cell-mediated autoimmunity.

The differentiation of Th17 cells is reciprocally related to that of Foxp $3^{+}$iTregs (50). TGF- $\beta$ induces Foxp 3 in naive CD 4 T cells, whereas IL- 6 and IL-21 suppress the TGF- $\beta$-driven induction of Foxp3, and TGF- $\beta$ plus IL-6 or IL-21 together induce ROR $\gamma$ t and the Th17 transcriptional program (31). At the transcription factor level, the balance between Th17 and iTregs is mediated by the antagonistic interaction of Foxp3 and ROR $\gamma$ t. Considering that miR-146a deficiency promotes Th17 differentiation, one may 


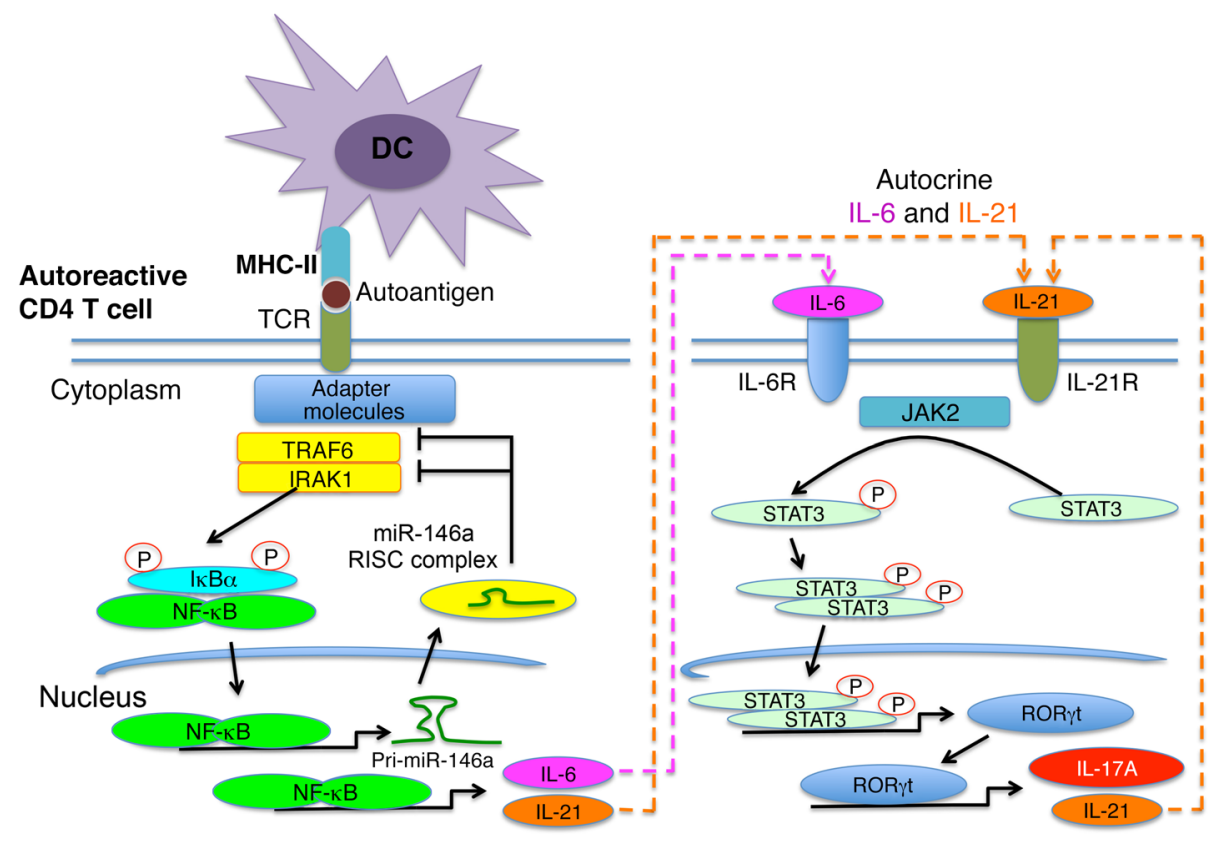

Figure 9. Schematic of an autocrine IL-6/IL-21 blockade model, in which miR-146a inhibits the development of pathogenic Th17 cells by blocking autocrine IL-6 and IL-21 signals in autoreactive CD4 T cells. Upon recognition of autoantigens, autoreactive CD4 T cells initiate TCR signaling that leads to NF- $K B$ activation. NF- $K B$ induces the production of autocrine IL- 6 and IL-21 cytokines, which activate STAT3. STAT3 induces the expression of the "master regulator" gene of Th17 cell differentiation, ROR $\gamma$ t, which then induces the expression of Th17 effector cytokines such as IL-17A, as well as more autocrine IL-21. Therefore, autocrine IL-6 and IL-21 promote the differentiation of autoreactive CD4 T cells into pathogenic Th17 cells. As a negative feedback regulator of NF- $\mathrm{KB}$ signaling, miR-146a is induced by NF- $\mathrm{KB}$, which in turn downregulates NF- $\kappa B$ activity through repression of the NF- $\kappa B$ signaling transducers TRAF6 and IRAK1. Through the downregulation of NF- $\mathrm{KB}$ activity, miR-146a blocks autocrine IL- 6 and IL-21 signals in autoreactive CD4 T cells and inhibits their differentiation into pathogenic Th17 cells. Pri-miR-146a, primary miR-146a; RISC, RNA-induced silencing complex.

predict that miR-146a deficiency inhibits the differentiation of iTregs. Indeed, in the 2D2 T cell-induced EAE model, we detected increased differentiation of Th17 cells and decreased differentiation of Foxp3 ${ }^{+}$iTregs when the 2D2 T cells were deficient in miR-146a (Figure 2, C and D, and Supplemental Figure 2, A and B). Furthermore, when we cultured 2D2 and 2D2-KO T cells in vitro in the presence of TGF- $\beta$, we observed a reduced induction of iTregs in 2D2-KO T cells (Supplemental Figure 2, C and D). Interestingly, one validated molecular target of miR-146a in autoreactive CD4 T cells, IRAK1, has been implicated in regulating the relative activity of STAT3 and NFAT signaling pathways in mouse CD4 $\mathrm{T}$ cells, and through that modulating the balance of their Th17 and iTreg differentiations (51). Therefore, miR-146a may regulate the induction of iTregs through modulation of the relative activities of both Foxp3/ROR $\gamma$ t and STAT3/NFAT signaling pathways. On the other hand, the total number of Foxp3 $3^{+}$ $\mathrm{CD} 4 \mathrm{~T}$ cells is increased in miR-146a-deficient mice compared with the total number in WT mice, although these Foxp $3^{+} \mathrm{CD} 4 \mathrm{~T}$ cells display impaired immune-suppressive function (22). Under steady-state conditions, the majority of Foxp $3^{+}$cells in mice are considered to be nTregs generated from the thymus, while nTregs follow a developmental path that may be different from that of iTregs (52). Hence, these results suggest that miR-146a may regulate nTregs and iTregs in different ways: for nTregs, miR-146a maintains their immune-suppressive function, while for iTregs, miR-146a promotes their differentiation from naive autoreactive $\mathrm{CD} 4 \mathrm{~T}$ cells. Aside from its role in the direct regulation of Tregs, we also examined whether miR-146a regulates autoreactive effector $\mathrm{T}$ cells in their response to Treg-mediated suppression. Using an in vitro Treg suppression assay, in which WT and miR-146a-deficient naive $\mathrm{CD} 4 \mathrm{~T}$ cells were stimulated in the presence of $\mathrm{GFP}^{+} \mathrm{Foxp}^{+}$Tregs isolated from Foxp3-GFP reporter mice, we found that miR-146a-deficient $\mathrm{CD} 4$ effector $\mathrm{T}$ cells were more resistant to Treg-mediated suppression (Supplemental Figure 2E). Overall, we found that miR-146a supports Treg-mediated suppression activities and prevents autoimmunity, both are examples of how miR-146a regulates the same physiological function through multiple pathways. It is likely that miR-146a regulates autoimmunity through many other mechanisms that are yet to be identified. Identification of these miR-146a-targeting cells, molecules, and signaling pathways could be an interesting direction for future research.

Dysregulated expression of miR146a has been correlated with an increased incidence of many human autoimmune diseases including MS, rheumatoid arthritis (RA), systemic lupus erythematosus (SLE), and type 1 diabetes (T1D) (53-55). GWAS also suggest that polymorphisms in miR-146a are associated with a susceptibility to autoimmune diseases like RA and SLE (56-58). Many autoimmune diseases are correlated with Th17 responses (59). In human clinical trials, inhibition of the IL-23/IL-17 inflammatory axis via the blocking of antibodies reduced the severity of psoriasis and RA (60). Together with this clinical evidence, our study suggests that miR-146a may be an attractive therapeutic target for treating a broad range of autoimmune diseases through the modulation of autoreactive $\mathrm{CD} 4 \mathrm{~T}$ cell $\mathrm{Th} 17$ responses.

\section{Methods}

Mice and materials. C57BL/6J (B6), B6.SJL-Ptprc $\mathrm{Pepc}^{\mathrm{b}}$ /BoyJ (CD45.1, BoyJ), B6.129S7-Rag1 $1^{\text {tm IMom }} / \mathrm{J}$ (RAG1-/), and C57BL/6-Tg(Tcra2D2, Tcrb2D2)1Kuch/J (2D2-Tg) mice were purchased from The Jackson Laboratory. miR-146a-deficient mice on a C57BL/6J background (miR$\left.146 a^{-/}\right)$were generated at the California Institute of Technology (20) and maintained at UCLA. C57BL/6-Tg(Foxp3-GFP)90Pkraj/J mice (Foxp3-GFP reporter mice) were provided by A. Ribas (UCLA). 2D2-Tg mice deficient in miR-146a (2D2/miR-146a/) were generated through breeding 2D2-Tg mice with $m i R-146 a^{-/-}$mice. Six- to ten-week-old female mice were used for all experiments unless otherwise indicated. 
$\mathrm{MOG}_{35-55}$, recognized by the 2D2-Tg T cells, was purchased from Hooke Laboratories. PMA and ionomycin were purchased from Calbiochem. GolgiStop was purchased from BD Biosciences. Recombinant mouse IL-4, IL-6, IL-12, IL-21, IL-23, and TGF- $\beta$ were purchased from PeproTech. The NF- $\mathrm{BB}$ inhibitor Bay 11-7082 was purchased from Sigma-Aldrich.

Antibodies and flow cytometry. Fluorochrome-conjugated monoclonal antibodies specific for mouse CD45.2 (catalog 109825; clone 104); TCR $\beta$ (catalog 109220; clone H57-597); CD4 (catalog 100531; clone GK1.5); IFN- $\gamma$ (catalog 505806; clone XMG1.2); and GM-CSF (catalog 505405; clone MP1-22E9) were purchased from BioLegend; and monoclonal antibodies specific for mouse IL-17A (catalog 25-717782; clone eBio17B7) and Foxp3 (catalog 12-5773-82; clone FJK-16s) were purchased from eBioscience. Fc block (anti-mouse CD16/32) (catalog 553142; clone 2.4G2) was purchased from BD Biosciences. Invitrogen eBioscience Fixable Viability Dye eFluor 506 (catalog 65-0866) was purchased from Thermo Fisher Scientific. Cells were stained with Fixable Viability Dye first, followed by Fc blocking and surface marker staining, using a standard, previously described procedure (61). To detect intracellular cytokines, cells were subjected to intracellular cytokine staining using a Cell Fixation/Permeabilization Kit (BD Biosciences) following the manufacturer's instructions. Stained cells were analyzed using a MACSQuant Analyzer 10 Flow Cytometer (Miltenyi Biotec). FlowJo software (Tree Star) was used to analyze the data.

LEAF purified anti-mouse CD3 $\varepsilon$ (catalog 100314; clone 145-2C11) and anti-mouse CD28 (catalog 102112; clone 37.51) antibodies, as well as neutralizing anti-mouse IL-4 (catalog 504115; clone 11B11); antimouse IFN- $\gamma$ (catalog 513206; clone XMG1.2); anti-mouse IL-12/IL-23 P40 (catalog 505202; clone C15.6); anti-IL-6 (catalog 504506; clone MP5-20F3); and isotype control antibodies used for in vitro culturing of mouse $\mathrm{T}$ cells were purchased from BioLegend. eBioscience functional-grade anti-mouse IL-21-neutralizing antibody (catalog 16-721185; clone FFA21) was purchased from Thermo Fisher Scientific.

EAE mouse model. EAE was induced in B6 WT and miR-146a ${ }^{-1-}$ mice using the EAE Induction Hooke Kit EK-2110 (Hooke Laboratories), according to the manufacturer's instructions. In brief, on day 0 , $\mathrm{MOG}_{35-55} / \mathrm{CFA}$ emulsion and PTX were injected. Each mouse received $200 \mu \mathrm{l}$ emulsion that contained $200 \mu \mathrm{g} \mathrm{MOG}_{35-55}$ and $400 \mathrm{ng}$ PTX. On day 1, a second dose of PTX (400 ng per mouse) was injected. After EAE induction, the mice were monitored daily for clinical symptoms of disease, and disease severity was scored regularly according to the following criteria: 0 , no disease; 1 , tail weakness or wobbly gait; 2 , hind limb paresis; 3 , hind limb paralysis; 4 , hind and forelimb paralysis; 5 , death. Paralyzed mice were given easy access to food and water.

At the various time points during EAE development, the experimental mice were euthanized for analysis. Spinal cords were harvested for the isolation of infiltrating CD4 T cells using Percoll gradient separation of lymphocytes followed by MACS sorting of CD $4^{+}$cells. The purified spinal cord-infiltrating CD4 T cells were then analyzed for gene expression using qPCR, or for Th17 cell presence using flow cytometry. For some experimental animals, splenic cells were harvested and cultured in vitro in a 24 -well plate at $2 \times 10^{6}$ cells per well, in the presence of $50 \mu \mathrm{g} / \mathrm{ml} \mathrm{MOG}_{35-55}$ for 4 days. Over the time course, cell culture supernatants were collected for ELISA analysis of Th cytokine production (IFN- $\gamma$, IL-4, and IL-17A).

Histological analysis. Spinal cords harvested from the experimental EAE mice were fixed with $10 \%$ neutral-buffered formalin and embedded in paraffin for sectioning (5- $\mu$ m thickness), followed by H\&E staining using standard procedures (UCLA Translational Pathology Core Laboratory). The sections were imaged using an Olympus BX51 upright microscope equipped with a SPOT insight CCD camera. EAE histology scores were assigned on the basis of a combination of previously published criteria $(62,63)$. Briefly, we used an ordinal scale scoring system: 0 , no inflammatory infiltrates; 1 , small and rare perivascular infiltrates; 2 , small but numerous perivascular infiltrates; 3 , numerous perivascular lesions and parenchymal infiltration; and 4, severe confluent inflammatory lesions. The samples were assessed by a pathologist (DSR) who was blinded to the sample group assignments during initial scoring.

Isolation of 2D2 autoreactive CD4 T cells. Splenic and lymph node cells were harvested from the 2D2-Tg and 2D2/miR-146 $a^{-/}$mice and then subjected to magnetic-activated cell sorting (MACS) using a Mouse Naive CD4 T Cell Isolation Kit (Miltenyi Biotec) according to the manufacturer's instructions. The resulting naive CD4 $\mathrm{T}$ cells specific for $\mathrm{MOG}_{35-55}$, denoted as 2D2 or 2D2-KO T cells, respectively, were then used for further in vivo and in vitro studies.

In vivo 2D2 T cell adoptive transfer EAE mouse model. Purified 2D2 or 2D2-KO T cells were adoptively transferred into $R A G 1^{-/-}$recipient mice via i.v. injection $\left(0.5 \times 10^{6}\right.$ to $1 \times 10^{6} \mathrm{~T}$ cells per recipient). The next day, the $R A G 1^{-/-}$recipient mice were subjected to EAE induction followed by evaluation using the same procedures and criteria as those described for the WT and $m i R-146 a^{-/}$EAE mouse model. Splenic cells were harvested after the experimental EAE $R A G 1^{-/-}$recipient mice were sacrificed. Some splenic cells were immediately used for intracellular cytokine staining, while other splenic cells were used for extended culturing. For intracellular cytokine straining, splenic cells were cultured for 5 hours in a 24 -well plate at $2 \times 10^{6}$ cells per well, in the presence of PMA (50 ng/ml) and ionomycin $(500 \mathrm{ng} / \mathrm{ml})$. GolgiStop ( $4 \mu \mathrm{l}$ per $6-\mathrm{ml}$ culture) was added to the culture for the last 4 hours. At the end of the culture, cells were collected and intracellular IL-17A cytokine staining was performed using a Cell Fixation and Permeabilization Kit (BD Biosciences). The cells were also costained with surface markers to identify 2D2 and 2D2-KO T cells (gated as TCR $\beta^{+} \mathrm{CD} 4^{+}$). For extended culturing, some splenic cells were cultured in a 24 -well plate at $2 \times 10^{6}$ cells per well in the presence of $50 \mu \mathrm{g} / \mathrm{ml} \mathrm{MOG}_{35-55}$ for 4 days, with the addition of irradiated ( $40 \mathrm{~Gy}$ ) B6 mouse splenic cells as antigen-presenting cells $\left(2 \times 10^{6}\right.$ cells per well). On day 4 , cell culture supernatants were collected for ELISA analysis of Th cytokine production (IFN- $\gamma$, IL-4, and IL-17A).

In vitro $2 \mathrm{D} 2 \mathrm{~T}$ cell activation assays. Naive $\mathrm{CD} 4 \mathrm{~T}$ cells sorted from WT and miR-146a-deficient 2D2-Tg mice (denoted as 2D2 and 2D2-KO T cells, respectively) were cultured in vitro in a 24 -well plate at $1 \times 10^{6}$ cells per well for up to 4 days, in the presence of plate-bound anti-CD3 $(5 \mu \mathrm{g} / \mathrm{ml})$ and anti-CD28 $(1 \mu \mathrm{g} / \mathrm{ml})$ as T cell stimulators. In some experiments, anti-IL-6-neutralizing $(10 \mu \mathrm{g} / \mathrm{ml})$ and/or antiIL-21-neutralizing $(10 \mu \mathrm{g} / \mathrm{ml})$ antibodies were added to the $\mathrm{T}$ cell culture to block IL-6 and/or IL-21 signaling, and the NF- $\kappa \mathrm{B}$ inhibitor Bay 11-7082 $(20 \mu \mathrm{M})$ was added to the $\mathrm{T}$ cell culture to block NF- $\mathrm{BB}$ signaling. At the desired time points, cell culture supernatants were collected for ELISA analysis of cytokine production, and cells were collected for analysis of miR or mRNA expression using qPCR or analysis of protein expression by Western blotting.

In vitro $2 \mathrm{D} 2 \mathrm{~T}$ cell Th differentiation assays. Naive $\mathrm{CD} 4 \mathrm{~T}$ cells sorted from WT and miR-146a-deficient 2D2-Tg mice (denoted as 2D2 and 
2D2-KO T cells, respectively) were cultured in vitro in a 24 -well plate at $1 \times 10^{6}$ cells per well for 4 days in the presence of plate-bound antiCD3 $(5 \mu \mathrm{g} / \mathrm{ml})$ and anti-CD28 $(1 \mu \mathrm{g} / \mathrm{ml})$ as T cell stimulators, with the following Th-polarizing additions: for ThO, no additional cytokines or neutralizing antibodies; for Th1, IL-12 (20 ng/ml) plus anti-IL-4 $(10 \mu \mathrm{g} / \mathrm{ml})$; for Th2, IL-4 (100 ng/ml) plus anti-IFN- $\gamma(10 \mu \mathrm{g} / \mathrm{ml})$ plus anti-IL-12/anti-IL-23 P40 (10 $\mu \mathrm{g} / \mathrm{ml})$; and for iTregs, TGF- $\beta$ ( $5 \mathrm{ng} / \mathrm{ml})$. For Th17 polarization, two culture conditions were used: one activated the classical IL-6/TGF- $\beta /$ ROR $\gamma$ t pathway through the addition of IL- 6 (50 ng/ml) plus TGF- $\beta$ (5 ng/ml) plus IL-23 (20 ng/ml), denoted as a Th17 (+IL-6) culture condition; while the other activated the alternative IL-21/TGF- $\beta$ /ROR $\gamma$ t pathway through the addition of IL-21 (100 $\mathrm{ng} / \mathrm{ml})$ plus TGF- $\beta$ ( $5 \mathrm{ng} / \mathrm{ml})$ plus IL-23 (20 ng/ml), denoted as a Th17 (+IL-21) culture condition. On day 4, cells were restimulated with PMA $(50 \mathrm{ng} / \mathrm{ml})$ plus ionomycin $(500 \mathrm{ng} / \mathrm{ml})$ for 4 hours with the addition of GolgiStop, and then analyzed for Th cytokine production using intracellular cytokine staining. In some experiments, cells were collected on day 4 and analyzed for mRNA expression using qPCR.

In vitro $2 \mathrm{D} 2 \mathrm{~T}$ cell retroviral transduction. The MIG and MIG-miR146a retroviral vectors have been described previously (23). Retroviruses were made using HEK293.T cells following a standard calcium precipitation method (64). Naive CD4 T cells isolated from WT or miR-146a-deficient 2D2-Tg mice were stimulated in vitro for 4 days and spin infected with retroviruses following a previously described standard procedure (23). On day 4 , the transduced $T$ cells were collected for direct analysis or follow-up assays.

In vitro $2 \mathrm{D} 2-\mathrm{KO} T$ cell siRNA transfection assays. Naive $\mathrm{CD} 4 \mathrm{~T}$ cells sorted from miR-146a-deficient 2D2-Tg mice (denoted as 2D2-KO T cells) were cultured in vitro in a 24 -well plate at $1 \times 10^{6}$ cells per well for 4 days in the presence of plate-bound anti-CD3 $(5 \mu \mathrm{g} / \mathrm{ml})$ and anti-CD28 $(1 \mu \mathrm{g} / \mathrm{ml})$ as $\mathrm{T}$ cell stimulators and under the desired Thpolarizing conditions. Cells were treated with $1 \mu \mathrm{M}$ Accell SMARTpool siRNAs specific for either mouse TRAF6 or mouse IRAK1 or with a nonsilencing control, according to the manufacturer's instructions (Thermo Fisher Scientific). On day 4, cells and cell culture supernatants were collected for analysis.

In vitro Treg suppression assay. Naive CD4 T cells (Tn cells) were sorted from WT and miR-146a-deficient mice (denoted as WT and $m i R-146 a^{-/-} \mathrm{T}$ cells, respectively) using a Mouse Naive CD4 ${ }^{+} \mathrm{T}$ Cell Isolation Kit (Miltenyi Biotec) according to the manufacturer's instructions. The WT and miR-146a $\mathrm{a}^{-1-}$ Tn cells were then cultured in vitro in a 96-well round-bottomed plate at $2 \times 10^{4}$ cells per well for 3 days, with the addition of titrated numbers of nTregs (at nTreg/Tn ratios of 1:1, $1: 2,1: 4,1: 8$, and $0: 1)$, and in the presence of soluble anti-CD3 $(1 \mu \mathrm{g} /$ $\mathrm{ml})$ and anti-CD28 $(1 \mu \mathrm{g} / \mathrm{ml})$ as $\mathrm{T}$ cell stimulators, as well as irradiated (20 Gy) BoyJ mouse splenic cells (CD45.2-) as antigen-presenting cells $\left(1 \times 10^{5}\right.$ cells per well). $n$ Tregs were sorted from the splenic and lymph node cells of Foxp3-GFP reporter mice using flow cytometry (gated as $\mathrm{TCR} \beta^{+} \mathrm{CD} 4^{+} \mathrm{GFP}^{+}$cells). On day 3 , the cell cultures were analyzed for the presence of effector CD4 $\mathrm{T}$ cells (gated as CD $45.2^{+} \mathrm{TCR} \beta^{+} \mathrm{CD} 4^{+}$ GFP $^{-}$cells) using flow cytometry.

ELISA. ELISAs to detect mouse cytokines were performed following a standard protocol from BD Biosciences. Capture and biotinylated antibody pairs for the detection of mouse IFN- $\gamma$, IL-4, IL-17A, and IL- 6 were also purchased from BD Biosciences. The streptavidin-HRP conjugate and mouse IFN- $\gamma$, IL-4, and IL-17A ELISA Ready-SET-Go (RSG) standard kits were purchased from Thermo Fisher Scientific
(eBioscience, Invitrogen). The 3,3',5,5'-tetramethylbenzidine (TMB) substrate was purchased from KPL. ELISAs for the detection of mouse IL-21 and soluble IL-6 R $\alpha$ (sIL-6R $\alpha$ ) were performed using a Mouse IL-21 or a Mouse IL-6 R $\alpha$ DuoSet Kit (R\&D Systems) following the manufacturer's instructions. Samples were analyzed for absorbance at $450 \mathrm{~nm}$ using an Infinite M1000 microplate reader (Tecan).

$m R N A$ qPCR. Total RNA was isolated using TRIzol Reagent (Invitrogen, Thermo Fisher Scientific) and an RNeasy Mini Kit (QIAGEN) according to the manufacturers' instructions. cDNA was prepared using a SuperScript III First-Strand Synthesis Supermix Kit (Invitrogen, Thermo Fisher Scientific). Gene expression was measured using a KAPA SYBR FAST qPCR Kit (Kapa Biosystems) and a 7500 Realtime PCR System (Applied Biosystems) according to the manufacturers' instructions. Ube2d 2 was used as an internal control because of its relative constant mRNA level during $T$ cell activation (65). The relative expression of the mRNA of interest was calculated using the $2^{\Delta \triangle C T}$ method and is presented as the fold induction relative to the control.

$m i R$ qPCR. miR qPCR was performed as previously described (23). In brief, total RNA was isolated using TRIzol Reagent (Invitrogen, Thermo Fisher Scientific) and an RNeasy Mini Kit (QIAGEN) following the manufacturers' instructions. miR-146a expression was measured using TaqMan miR assays (Applied Biosystems) and a 7500 Real-time PCR System (Applied Biosystems) according to the manufacturers' instructions. snoRNA2O2 was used as an internal control. The relative expression of miR-146a was calculated using the $2^{\Delta \Delta C T}$ method and is presented as the fold induction relative to the control.

Western blot analysis. Total protein was extracted using a lysis buffer containing 20 mM HEPES (pH 7.6), 150 mM NaCl, 1 mM EDTA; $1 \%$ TritonX-100, a phosphatase inhibitor cocktail (Sigma-Aldrich), and a protease inhibitor cocktail (Roche). Nuclear protein was extracted using a Nuclear Protein Extraction Kit (Sigma-Aldrich). Protein concentration was measured using the Bradford assay reagent (Bio-Rad). Equal amounts of protein were resolved on a 10\% SDS-PAGE gel and then transferred to a nitrocellulose membrane by electrophoresis. The following antibodies were purchased from Cell Signaling Technology and used to blot for the protein of interest: anti-mouse p-STAT3 (catalog 4904S; clone S727); anti-mouse STAT3 (catalog 9134S; clone 79D7); anti-mouse NF-кB p65 (catalog 8242P; clone D14E12); antimouse NF-кB cRel (catalog 12707S; clone D6Y6M); anti-mouse IRAK1 (catalog 4504S; clone D51G7); anti-mouse p-SHP2 (catalog 3397T; clone D50F2); anti-mouse SHP2 (catalog 3751T; clone Y542); antimouse SOCS3 (catalog 2923S); and anti-mouse gp130 (catalog 3732S). Anti-mouse TRAF6 was purchased from MBL (catalog 597). $\beta$-Actin (Santa Cruz Biotechnology Inc.; catalog sc-69879; clone AC-15) was used as an internal control for total protein extracts, while lamin A (Santa Cruz Biotechnology Inc.; catalog sc-71481; clone 4A58) was used as an internal control for nuclear protein extracts. Signals were visualized with autoradiography using an ECL system (Thermo Fisher Scientific). Data analysis was performed using ImageJ software (NIH).

Microarray analysis. Naive CD4 T cells sorted from WT and miR146a-deficient 2D2-Tg mice (denoted as 2D2 and 2D2-KO T cells, respectively) were cultured in vitro in a 24 -well plate at $1 \times 10^{6}$ cells per well for 3 days, in the presence of plate-bound anti-CD3 $(5 \mu \mathrm{g} /$ $\mathrm{ml})$ and anti-CD28 $(1 \mu \mathrm{g} / \mathrm{ml})$. On day 3 , cells were collected, and total RNA was isolated for whole-genome gene expression profile analysis using the GeneChip Mouse Genome 430 2.0 Arrays, following a standard Affymetrix microarray protocol (UCLA Clinical Microar- 
ray Core). Biotinylated cRNA was prepared from 100 ng total RNA. Following fragmentation, $10 \mu \mathrm{g}$ cRNA was hybridized for 16 hours at $45^{\circ} \mathrm{C}$ on the microarray. GeneChips were washed and stained in the Affymetrix Fluidics Station 450 and then scanned using the GeneChip Scanner 3000 7G. Gene expression level analysis and normalization were performed using the Affymetrix Microarray Suite 5 (mas5.0) method and Affymetrix Transcriptome Analysis Console (TAC) 2.0 software. ANOVA was used to calculate the $P$ value. Genes showing altered expression with a $P$ value of less than 0.05 and greater than 1.5 fold changes were considered differentially expressed. The heatmap was generated using Excel software to show the differential expression of the selected genes. Ingenuity Pathway Analysis (IPA) software (QIAGEN) was used to conduct pathway analysis.

Statistics. Pairwise comparisons were made using a 2-tailed Student's $t$ test. Multiple comparisons were performed using an ordinary 1-way or 2-way ANOVA followed by Tukey's multiple comparisons test. Data are presented as the mean \pm SEM, unless otherwise indicated. A $P$ value of less than 0.05 was considered significant. GraphPad Prism 6 (GraphPad Software) was used for all statistical analyses.

Study approval. All animal experiments were performed according to protocols approved by the IACUC of UCLA.

Accession numbers. Microarray data shown in this study have been deposited in the NCBI's Gene Expression Omnibus (GEO) database (https://www.ncbi.nlm.nih.gov/geo/) under the accession number GSE101165.

\section{Author contributions}

$\mathrm{BL}, \mathrm{XW}$, and LY designed the experiments, conducted experiments, analyzed data, and wrote the manuscript. IYC, YCW, SL, and DSR conducted experiments and analyzed data. ATP, HM, and DJS conducted experiments. MPB analyzed the data.

\section{Acknowledgments}

We thank the UCLA animal facility for providing animal support; the UCLA Translational Pathology Core Laboratory (TPCL) for providing histology support; the UCLA Clinical Microarray Core for providing microarray support; the Laboratory of Antoni Ribas (UCLA) for providing Foxp3-GFP reporter mice; the Laboratory of Genhong Cheng (UCLA) for providing assistance on EAE mouse model; and Pin Wang (University of Southern California, Los Angeles, California, USA) for critical reading of this manuscript. This work was supported by a UCLA New Faculty Startup grant (to LY); a Director's New Innovator Award from the NIH (DP2 CA196335, to LY); and a grant from the NIH (R01 CA166450, to DSR).

Address correspondence to: Lili Yang, Department of Microbiology, Immunology and Molecular Genetics, M/C 957243, Eli and Edythe Broad Center of Regenerative Medicine and Stem Cell Research, 610 Charles E. Young Drive East, TLSB 3032, UCLA, Los Angeles, California 90095, USA. Phone: 310.825.8609; Email: liliyang@ucla.edu.
1. Mosmann TR, Coffman RL. TH1 and TH2 cells: different patterns of lymphokine secretion lead to different functional properties. Annu Rev Immunol. 1989;7:145-173.

2. Zhou L, Chong MM, Littman DR. Plasticity of CD4+ T cell lineage differentiation. Immunity. 2009;30(5):646-655.

3. Locksley RM. Nine lives: plasticity among T helper cell subsets. J Exp Med. 2009;206(8):1643-1646.

4. Murphy KM, Stockinger B. Effector T cell plasticity: flexibility in the face of changing circumstances. Nat Immunol. 2010;11(8):674-680.

5. Murphy KM, Reiner SL. The lineage decisions of helper T cells. Nat Rev Immunol. 2002;2(12):933-944.

6. Schmitt E, Bopp T. Discovery and initial characterization of Th9 cells: the early years. Semin Immunopathol. 2017;39(1):5-10.

7. Azizi G, Yazdani R, Mirshafiey A. Th22 cells in autoimmunity: a review of current knowledge. Eur Ann Allergy Clin Immunol. 2015;47(4):108-117.

8. Zhu J, Yamane H, Paul WE. Differentiation of effector CD4 T cell populations (*). Annu Rev Immunol. 2010;28:445-489.

9. O'Shea JJ, Paul WE. Mechanisms underlying lineage commitment and plasticity of helper CD4+ T cells. Science. 2010;327(5969):1098-1102.

10. Wilson CB, Rowell E, Sekimata M. Epigenetic control of T-helper-cell differentiation. Nat Rev Immunol. 2009;9(2):91-105.

11. Kanno Y, Vahedi G, Hirahara K, Singleton K, O'Shea JJ. Transcriptional and epigenetic control of T helper cell specification: molecular mechanisms underlying commitment and plasticity. Annu Rev Immunol. 2012;30:707-731.

12. Korn T, Bettelli E, Oukka M, Kuchroo VK.
IL-17 and Th17 Cells. Annu Rev Immunol. 2009;27:485-517.

13. Dong C. TH17 cells in development: an updated view of their molecular identity and genetic programming. Nat Rev Immunol. 2008;8(5):337-348.

14. Ambros V. The functions of animal microRNAs. Nature. 2004;431(7006):350-355.

15. Bartel DP. MicroRNAs: genomics, biogenesis, mechanism, and function. Cell. 2004;116(2):281-297.

16. Baltimore D, Boldin MP, O'Connell RM, Rao DS, Taganov KD. MicroRNAs: new regulators of immune cell development and function. Nat Immunol. 2008;9(8):839-845.

17. Tsitsiou E, Lindsay MA. microRNAs and the immune response. Curr Opin Pharmacol. 2009;9(4):514-520.

18. O'Connell RM, Rao DS, Baltimore D. microRNA regulation of inflammatory responses. Annu Rev Immunol. 2012;30:295-312.

19. Taganov KD, Boldin MP, Chang KJ, Baltimore D. NF-kappaB-dependent induction of microRNA miR-146, an inhibitor targeted to signaling proteins of innate immune responses. Proc Natl Acad Sci U S A. 2006;103(33):12481-12486.

20. Boldin MP, et al. miR-146a is a significant brake on autoimmunity, myeloproliferation, and cancer in mice. J Exp Med. 2011;208(6):1189-1201.

21. Zhao JL, Rao DS, Boldin MP, Taganov KD, O'Connell RM, Baltimore D. NF-kappaB dysregulation in microRNA-146a-deficient mice drives the development of myeloid malignancies. Proc Natl Acad Sci U S A. 2011;108(22):9184-9189.

22. Lu LF, et al. Function of miR-146a in controlling Treg cell-mediated regulation of Th1 responses. Cell. 2010;142(6):914-929.
23. Yang L, et al. miR-146a controls the resolution of T cell responses in mice. J Exp Med. 2012;209(9):1655-1670.

24. Hafler DA. Multiple sclerosis. J Clin Invest. 2004;113(6):788-794.

25. Bettelli E, Pagany M, Weiner HL, Linington C, Sobel RA, Kuchroo VK. Myelin oligodendrocyte glycoprotein-specific $\mathrm{T}$ cell receptor transgenic mice develop spontaneous autoimmune optic neuritis. J Exp Med. 2003;197(9):1073-1081.

26. Ivanov II, et al. The orphan nuclear receptor RORgammat directs the differentiation program of proinflammatory IL-17+ T helper cells. Cell. 2006;126(6):1121-1133.

27. Veldhoen M, Hocking RJ, Atkins CJ, Locksley RM, Stockinger B. TGFbeta in the context of an inflammatory cytokine milieu supports de novo differentiation of IL-17-producing T cells. Immunity. 2006;24(2):179-189.

28. Zhou L, et al. IL-6 programs T(H)-17 cell differentiation by promoting sequential engagement of the IL-21 and IL-23 pathways. Nat Immunol. 2007;8(9):967-974.

29. Kishimoto T, Akira S, Taga T. Interleukin-6 and its receptor: a paradigm for cytokines. Science. 1992;258(5082):593-597.

30. Oeckinghaus A, Hayden MS, Ghosh S. Crosstalk in NF- $\kappa$ B signaling pathways. Nat Immunol. 2011;12(8):695-708.

31. Bettelli E, et al. Reciprocal developmental pathways for the generation of pathogenic effector TH17 and regulatory T cells. Nature. 2006;441(7090):235-238.

32. Mangan PR, et al. Transforming growth factor-beta induces development of the $\mathrm{T}(\mathrm{H}) 17$ lineage. Nature. 2006;441(7090):231-234. 
33. Nurieva R, et al. Essential autocrine regulation by IL-21 in the generation of inflammatory T cells. Nature. 2007;448(7152):480-483.

34. Langrish CL, et al. IL-23 drives a pathogenic T cell population that induces autoimmune inflammation. JExp Med. 2005;201(2):233-240.

35. Samoilova EB, Horton JL, Hilliard B, Liu TS, Chen Y. IL-6-deficient mice are resistant to experimental autoimmune encephalomyelitis: roles of IL- 6 in the activation and differentiation of autoreactive T cells. J Immunol. 1998;161(12):6480-6486.

36. Okuda Y, et al. IL-6-deficient mice are resistant to the induction of experimental autoimmune encephalomyelitis provoked by myelin oligodendrocyte glycoprotein. Int Immunol. 1998;10(5):703-708.

37. Mendel I, Katz A, Kozak N, Ben-Nun A, Revel M. Interleukin- 6 functions in autoimmune encephalomyelitis: a study in gene-targeted mice. Eur J Immunol.1998;28(5):1727-1737.

38. Eugster HP, Frei K, Kopf M, Lassmann H, Fontana A. IL-6-deficient mice resist myelin oligodendrocyte glycoprotein-induced autoimmune encephalomyelitis. Eur J Immunol. 1998;28(7):2178-2187.

39. Van Snick J. Interleukin-6: an overview. Annu Rev Immunol. 1990;8:253-278.

40. Kishimoto T. Interleukin-6: from basic science to medicine-- 40 years in immunology. Annu Rev Immunol. 2005;23:1-21.

41. Libermann TA, Baltimore D. Activation of interleukin-6 gene expression through the NF-kappa B transcription factor. Mol Cell Biol. 1990;10(5):2327-2334

42. Du C, et al. MicroRNA miR-326 regulates TH-17 differentiation and is associated with the pathogenesis of multiple sclerosis. Nat Immunol. 2009;10(12):1252-1259.

43. Takahashi $\mathrm{H}$, et al. TGF- $\beta$ and retinoic acid induce the microRNA miR-10a, which targets Bcl-6 and constrains the plasticity of helper T cells. Nat Immunol. 2012;13(6):587-595.
44. O'Connell RM, et al. MicroRNA-155 promotes autoimmune inflammation by enhancing inflammatory T cell development. Immunity. 2010;33(4):607-619.

45. Murugaiyan G, Beynon V, Mittal A, Joller N, Weiner HL. Silencing microRNA-155 ameliorates experimental autoimmune encephalomyelitis. J Immunol. 2011;187(5):2213-2221.

46. Mycko MP, Cichalewska M, Machlanska A, Cwiklinska H, Mariasiewicz M, Selmaj KW. MicroRNA301a regulation of a T-helper 17 immune response controls autoimmune demyelination. Proc Natl Acad Sci U S A. 2012;109(20):E1248-E1257.

47. Baumjohann D, Ansel KM. MicroRNA-mediated regulation of $\mathrm{T}$ helper cell differentiation and plasticity. Nat Rev Immunol. 2013;13(9):666-678.

48. Curtale G, et al. An emerging player in the adaptive immune response: microRNA-146a is a modulator of IL-2 expression and activationinduced cell death in T lymphocytes. Blood. 2010;115(2):265-273.

49. Möhnle P, et al. MicroRNA-146a controls Th1cell differentiation of human CD4+ T lymphocytes by targeting PRKCe. Eur J Immunol. 2015;45(1):260-272.

50. Kimura A, Kishimoto T. IL-6: regulator of Treg/Th17 balance. Eur J Immunol. 2010;40(7):1830-1835.

51. Maitra U, Davis S, Reilly CM, Li L. Differential regulation of Foxp3 and IL-17 expression in CD4 T helper cells by IRAK-1. J Immunol. 2009;182(9):5763-5769.

52. Bilate AM, Lafaille JJ. Induced CD4+Foxp3+ regulatory T cells in immune tolerance. Annu Rev Immunol. 2012;30:733-758.

53. Garo LP, Murugaiyan G. Contribution of microRNAs to autoimmune diseases. Cell Mol Life Sci. 2016;73(10):2041-2051.

54. Rusca N, Monticelli S. MiR-146a in immunity and disease. Mol Biol Int. 2011;2011:437301.

55. Xu WD, Lu MM, Pan HF, Ye DQ. Association of microRNA-146a with autoimmune diseases. Inflammation. 2012;35(4):1525-1529.
56. Chatzikyriakidou A, Voulgari PV, Georgiou I, Drosos AA. A polymorphism in the 3'-UTR of interleukin-1 receptor-associated kinase (IRAK1), a target gene of miR-146a, is associated with rheumatoid arthritis susceptibility. Joint Bone Spine. 2010;77(5):411-413.

57. Chatzikyriakidou A, Voulgari PV, Georgiou I, Drosos AA. miRNAs and related polymorphisms in rheumatoid arthritis susceptibility. Autoimmun Rev. 2012;11(9):636-641.

58. Luo $\mathrm{X}$, et al. A functional variant in microRNA146a promoter modulates its expression and confers disease risk for systemic lupus erythematosus. PLoS Genet. 2011;7(6):e1002128.

59. Patel DD, Kuchroo VK. Th17 cell pathway in human immunity: Lessons from genetics and therapeutic interventions. Immunity. 2015;43(6):1040-1051.

60. Steinman L. Mixed results with modulation of $\mathrm{TH}-17$ cells in human autoimmune diseases. Nat Immunol. 2010;11(1):41-44.

61. Smith DJ, et al. Genetic engineering of hematopoietic stem cells to generate invariant natural killer T cells. Proc Natl Acad Sci U S A. 2015;112(5):1523-1528.

62. Racke MK, et al. Retinoid treatment of experimental allergic encephalomyelitis. IL-4 production correlates with improved disease course. J Immunol. 1995;154(1):450-458.

63. Langer-Gould A, Garren H, Slansky A, Ruiz PJ, Steinman L. Late pregnancy suppresses relapses in experimental autoimmune encephalomyelitis: evidence for a suppressive pregnancy-related serum factor. J Immunol. 2002;169(2):1084-1091.

64. Yang L, Baltimore D. Long-term in vivo provision of antigen-specific T cell immunity by programming hematopoietic stem cells. Proc Natl Acad Sci US A. 2005;102(12):4518-4523.

65. Hamalainen HK, et al. Identification and validation of endogenous reference genes for expression profiling of $\mathrm{T}$ helper cell differentiation by quantitative real-time RT-PCR. Anal Biochem. 2001;299(1):63-70. 